\title{
Paleomadrigueras de roedores, un nuevo método para el estudio del Cuaternario en zonas áridas de Sudamérica
}

\author{
Rodent middens, a new method for Quaternary research in arid zones \\ of South America
}

\author{
JULIO L. BETANCOURT ${ }^{1} \&$ BÁRBARA SAAVEDRA ${ }^{2}$ \\ ${ }^{1}$ Desert Laboratory, United States Geological Survey and University of Arizona, 1675 W Anklam Road, \\ Tucson, Arizona 85745, U.S.A.; e-mail jlbetanc@usgs.gov \\ ${ }^{2}$ Departamento de Ciencias Ecológicas, Facultad de Ciencias, Universidad de Chile, \\ Casilla 653, Santiago, Chile
}

\begin{abstract}
RESUMEN
Las zonas áridas y semiáridas de Sudamérica carecen de registros históricos exhaustivos de vegetación y clima, a pesar de la utilidad que ellos representan para el establecimiento de condiciones basales y tasas naturales de variabilidad en procesos abióticos y bióticos. Fuentes comúnmente utilizadas en el estudio de paleovegetación como el polen, son escasas en zonas áridas y semiáridas en Sudamérica, lo que se refleja en el limitado número de estudios vegetacionales durante el Cuaternario Tardío. El vacío existente en el conocimiento de la historia vegetacional de esta zona podría ser remediado gracias al descubrimiento y análisis de paleomadrigueras de roedores en ambientes rocosos. Estos depósitos, producidos por roedores de los géneros Lagidium, Phyllotis, Abrocoma y Octodontomys y posiblemente otros, son ricos en restos vegetales como ramas, hojas, polen o cutículas; y restos animales como fecas, huesos o insectos. Las paleomadrigueras de roedores han sido extensamente utilizadas en el estudio de zonas áridas en Norteamérica, donde más de 2.500 registros producidos por roedores del género Neotoma han sido analizadas desde 1960, permitiendo reconstruir una detallada historia de cambios vegetacionales y climáticos de los últimos 40.000 años en el suroeste de Norteamérica. Investigaciones recientes han revelado la presencia de paleomadrigueras en la pre-puna, los desiertos del Monte y Patagonia del oeste argentino, el desierto de Atacama al norte de Chile y sur del Perú, el matorral Mediterráneo de Chile central, y la Puna del Altiplano Andino. Estos hallazgos fortalecen el gran potencial que dichos depósitos tienen para reconstruir la vegetación y el clima en Sudamérica. Con el fin de aportar elementos para la detección, uso y análisis de paleomadrigueras de roedores, entregamos una síntesis de los depósitos registrados hasta la fecha en Sudamérica, así como una descripción de sus probables agentes formadores. Basados en la experiencia Norteamericana entregamos además, un resumen de los métodos existentes para la detección y análisis de dichos depósitos, los sesgos asociados, así como el aporte potencial que su análisis podría representar en futuros estudios paleoecológicos en las zonas áridas y semiáridas de Sudamérica.
\end{abstract}

Palabras clave: paleomadrigueras, roedores, paleovegetación, zonas áridas, Sudamérica.

\begin{abstract}
In arid and semi-arid regions of South America, historical evidence for climate and vegetation change is scarce despite its importance for determining reference conditions and rates of natural variability in areas susceptible to modern desertification. Normal lines of evidence, such as pollen stratigraphies from lakes, are either rare or unobtainable in deserts; studies of late Quaternary vegetation history are few and generally inconclusive. This gap in knowledge may be corrected with discovery and development of fossil rodent middens in rocky environments throughout arid South America. These middens, mostly the work of Lagidium, Phyllotis, Abrocoma and Octodontomys, are rich in readily identifiable plant macrofossils, cuticles and pollen, as well as vertebrate and insect remains. In the North American deserts, more than 2,500 woodrat (Neotoma) middens analyzed since 1960 have yielded a detailed history of environmental change during the past 40,000 years. Preliminary work in the pre-puna, Monte and Patagonian Deserts of western Argentina, the Atacama Desert of northern Chile/southern Peru, the Mediterranean matorral of central Chile, and the Puna of the Andean altiplano suggest a similar potential for rodent middens in South America. Here we borrow from the North American experience to synthesize methodologies and approaches, summarize preliminary work, and explore the potential of rodent midden research in South America.
\end{abstract}

Key words: middens, rodents, arid zones, South America. 


\section{INTRODUCCIÓN}

Las zonas áridas y semiáridas de nuestro planeta desempeñan un rol significativo en diversos procesos globales, tanto en el ámbito físico como socioeconómico. Estas zonas son especialmente sensibles a cambios climáticos de diferente intensidad. Sin embargo, muchas de estas áreas, especialmente en Sudamérica, carecen de registros históricos exhaustivos de vegetación y clima, a pesar de la utilidad que ellos tienen para el establecimiento de condiciones basales en procesos abióticos y bióticos, así como sus tasas naturales de variabilidad. Los registros de vegetación y clima de larga data son extremadamente útiles para predecir el sentido y tasas de cambio en procesos ecosistémicos sometidos a perturbación. Asimismo, registros históricos precisos permiten conocer los mecanismos que han generado variaciones climáticas en el pasado, aportando elementos significativos para la predicción de cambios similares en el futuro.

Los estudios de polen, fuente normalmente utilizada en estudios de paleovegetación, son escasos en zonas áridas y semiáridas en Sudamérica (Markgraf 1983). Ello se debe en parte, a la carencia de sedimentación húmeda continua, así como a la dominancia de especies vegetales polinizadas por insectos. Igualmente, la escasa preservación de granos de polen en sedimentos alcalinos frecuentes en dichas zonas, dificulta la formación de registros polínicos. Estos factores determinan por ejemplo, que taxa como Prosopis y Larrea, habituales en zonas áridas de Sudamérica, encuentren escasa representación en registros polínicos, a pesar de que ellas dominan la vegetación a nivel local.

Hasta hace poco, estudios de vegetación que describan el Cuaternario Tardío en Sudamérica se limitaron a: un registro polínico de 24.000 años en Laguna Junin $\left(11^{\circ} \mathrm{S}\right)$ en Perú central (Hansen et al. 1984); una estratigrafía polínica de 20.000 años en el Lago Titicaca, Bolivia (Ybert 1992); registros de 6.000-4.000 años en pantanos de la precordillera en la Provincia de Mendoza (Markgraf 1983); un registro de 30.000 años en sedimentos de la cueva Gruta del Indio, cerca de San Rafael, Mendoza (D'Antoni 1983); perfiles de 12.000 y 4.000 años en pantanos de Quereo y Quintero, respectivamente, en la costa central de Chile (Villagrán \& Varela 1990, Villa-Martínez \& Villagrán 1997); un perfil de 45.000 años en la laguna de Tagua Tagua en la precordillera de los Andes de Chile central (Heusser 1983); y un perfil de 11.000 años de Vaca Lauquén en la Provincia de Neuquén, Argentina (Markgraf 1987). Asombrosamente, Gruta del Indio es la única fuente de información paleoecológica que explora la transición Pleistoceno-Holoceno en el desierto del Monte, un área de tamaño equivalente al desierto de Sonora o Chihuahua.

Afortunadamente, la pobreza de registros paleobotánicos en zonas áridas ha sido superada gracias al descubrimiento de registros paleobotánicos en depósitos cavernosos (Betancourt et al. 1990a, Betancourt et al. 2000a, Latorre et al. en prensa, C. Latorre, J.L. Betancourt, K.A. Rylander, J. Quade \& O. Matthei resultados no publicados). Estos depósitos corresponden a paleomadrigueras ("middens"), las cuales son producidas por una variedad de animales, fundamentalmente roedores. Ellos son capaces de generar acumulación de material biológico en sus madrigueras, las cuales son ricas en restos vegetales (e.g., ramas, hojas, polen, cutículas) y animales (e.g., fecas, huesos, insectos). Al evaporarse la orina del roedor, estas acumulaciones se encapsulan, permitiendo la conservación de los materiales depositados, los cuales son capaces de permanecer intactos durante milenios.

Las paleomadrigueras de roedores han sido ampliamente utilizadas en el estudio de zonas áridas en Norteamérica (Betancourt et al. 1990a). Allí los depósitos son producidos exclusivamente por especies del género Neotoma y Erethizon dorsatum. Dicho material ha permitido la reconstrucción precisa de la vegetación y clima en desiertos del oeste de Norteamérica (e.g., Sonora, Mojave y Chihuahua) durante los últimos 40.000 años (ver síntesis Van Devender \& Spaulding 1979, Van Devender et al. 1987, Betancourt et al. 1990b, Thompson et al. 1993, Rhode 2001). El estudio de paleomadrigueras ha facilitado además, el conocimiento de diversos aspectos relacionados con la geología y geomorfología de estos sistemas desérticos.

Afortunadamente, la presencia de roedores con capacidad de generar paleomadrigueras no se restringe a las zonas áridas de América del Norte, sino que se extiende a otras áreas de escasa humedad, distribuidas en el resto de planeta. Ello ha permitido el desarrollo de estudios paleoecológicos en zonas áridas del Cercano Oriente (Fall 1990, Fall et al. 1990), África (Scott \& Vogel 1992, Thinon et al. 1996, Carrion et al. 1999), Asia central (Jason Rech, comunicación personal), Australia (Green et al. 1983, Nelson et al. 1990, Berry 1991, Pearson \& Dodson 1993), y Sudamérica (Barcena \& Roig 1982, Pearson \& Christie 1993, Fernández 1994, Braun \& Mares 1996, Markgraf et al. 1997, Betancourt et al. 2000a, Latorre et al. en prensa, C. Latorre, J.L. Betancourt, K.A. Rylander, J. Quade \& O. Matthei resultados no publicados). 
Investigaciones recientes han revelado la presencia de paleomadrigueras, similares a las producidas por roedores del género Neotoma, en zonas áridas y semiáridas de Sudamérica (Pearson \& Christie 1993, Braun \& Mares 1996, Markgraf et al. 1997, Betancourt et al. 2000a, Holmgren et al. 2001, Latorre et al. en prensa, C. Latorre, J.L. Betancourt, K.A. Rylander, J. Quade \& O. Matthei resultados no publicados, J.L. Betancourt resultados no publicados) (Fig. 1). Estos descubrimientos sugieren gran potencial en el uso de paleomadrigueras para reconstruir vegetación y clima en Sudamérica, en áreas como la Puna del Altiplano de Perú, Bolivia, Argentina y Chile; el Cardonal o Formación de Sierra al pie de la Cordillera Occidental de los Andes en el sur de Perú y norte de Chile; el Desierto Atacama del norte de Chile, sur de Perú; el límite entre desierto semiárido y matorral Mediterráneo en Chile central; el área de la pre-puna del noroeste de Argentina; el Monte y formaciones adyacentes en el oeste de Argentina, y la estepa patagónica del sur de Chile y Argentina.

El análisis de paleomadrigueras presenta ventajas respecto a otros métodos de reconstrucción paleoambiental. Por ejemplo, los granos de polen presentes en lagos u otros sedimentos, rara vez pueden ser identificados a niveles taxonómicos inferiores al género. Por el contrario, los macrorestos vegetales contenidos en paleomadrigueras usualmente pueden ser determinados hasta un nivel de especie. El supuesto de contemporaneidad y simpatría de los taxa presentes en depósitos de polen puede ser puesto a prueba en los ensambles fósiles de macrorestos vegetales, a través de la datación de especímenes individuales. Asimismo, el problema de la simpatría de los paleorestos vegetales inherente a los estudios de polen, se soluciona al utilizar las paleomadrigueras, por cuanto los macrofósiles vegetales presentes en cada depósito provienen de las inmediaciones de la madriguera. Las desventajas de las paleomadrigueras reconocidas hasta el momento, incluyen la falta de conocimiento de la duración del episodio deposicional, el cual no puede ser resuelto por fechado radiocarbónico. Una paleomadriguera puede representar desde meses hasta cientos de años de acumulación. A diferencia de los depósitos sedimentarios de lagos, la depositación de paleomadrigueras es discontinua. Ello dificulta las comparaciones temporales. Asimismo, la preservación de las paleomadrigueras se restringe a ambientes rocosos, por lo que la reconstrucción de paleovegetación no incorpora terrenos abiertos o de suelos más profundos (Davis 1990). A pesar de estos sesgos, las paleomadrigueras de roedores ofrecen una oportunidad enorme para la reconstrucción paleoambiental en regiones áridas y semiáridas, donde normalmente otros métodos no dan buenos resultados.

Con el fin de aportar elementos para la detección, uso y análisis de paleomadrigueras de roedores, entregamos aquí una síntesis de los depósitos registrados hasta la fecha en Sudamérica, así como una descripción de sus probables agentes formadores. Entregamos además, una síntesis de los métodos existentes para la detección y análisis de dichos depósitos, los sesgos asociados, así como el aporte potencial que su análisis podría representar en futuros estudios paleoecológicos en las zonas áridas y semiáridas de Sudamérica.

\section{ESPECIES FORMADORAS DE PALEOMADRIGUERAS}

Los roedores del género Neotoma poseen atributos particulares que favorecen la formación de paleomadrigueras. Son animales terrestres,

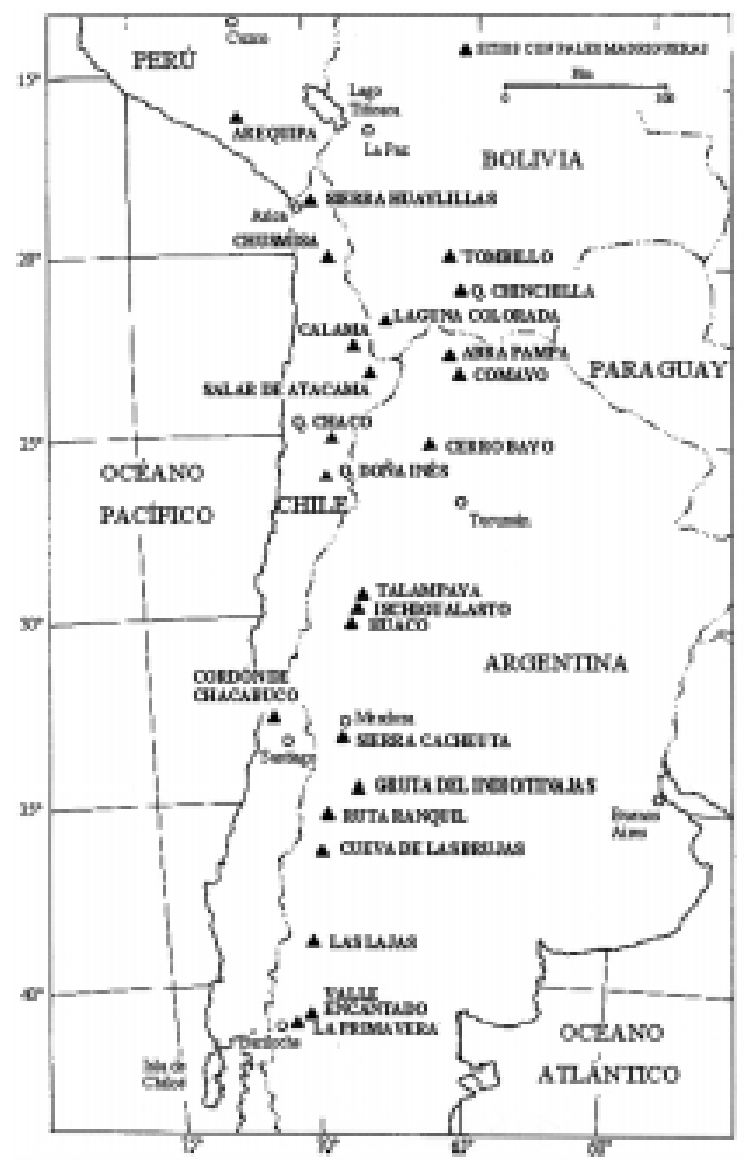

Fig. 1: Localización geográfica de zonas en las que se ha registrado la existencia de paleomadrigueras en Sudamérica.

Geographic localization of areas in which rodent middens have been collected in South America. 
escansoriales, buenos escaladores, y tienen cierta capacidad de cavar, no estando especializados en la vida fosorial ni arbórea. Presentan actividad nocturna y son herbívoros. Dichos taxa poseen la capacidad de acumular alimento. Tienen un modo de vida centrado en madrigueras, con ámbitos de hogar pequeños y marcada territorialidad. Son animales asociales, y cada madriguera es utilizada sólo por un individuo. Sin embargo, viven cercanos unos de otros, y cuando la calidad del hábitat lo permite, forman asociaciones numerosas de individuos. Las diferentes especies que conforman este género presentan diferente grado de especificidad trófica (Finley 1990).

En Sudamérica, a diferencia de lo observado en zonas áridas de Norteamérica, diversos géneros de roedores tendrían la capacidad de generar paleomadrigueras (Tabla 1). Contrario a lo obser-

TABLA 1

Taxa de roedores que generan o son potenciales responsables de la acumulación de paleomadrigueras en zonas áridas del sur de Sudamérica. Basado en Nowak \& Paradiso (1983), Ojeda \& Mares (1989), Redford \& Eisenberg (1992), Marquet et al. (1993), Wilson \& Reeder (1993), Anderson (1997), Steppan (1998)

Taxa that generate or could produce middens in arid zones of southern South America. Based on Marquet et al. (1993), Nowak \& Paradiso (1983), Ojeda \& Mares (1989), Redford \& Eisenberg (1992), Anderson (1997), Steppan (1998), Wilson \& Reeder (1993)

\begin{tabular}{|c|c|c|}
\hline Género & Especie & Distribución \\
\hline \multirow[t]{11}{*}{ Phyllotis } & amicus & Costa y pendientes suaves del oeste del Perú \\
\hline & caprinus & $\begin{array}{l}\text { Sur de Bolivia y en la vertiente oriental de Los Andes del norte de } \\
\text { Argentina. Chuquisaca, Potosi, Tarija }\end{array}$ \\
\hline & darwini & $\begin{array}{l}\text { Centro del Perú hasta el centro y sur de Chile. En Argentina ocupa la } \\
\text { vertiente oriental de Los Andes desde el sur de Mendoza hasta Buenos } \\
\text { Aires. Alcanza Tierra del Fuego }\end{array}$ \\
\hline & definitus & Andes de Ancash en Perú \\
\hline & gerbillus & Desierto de Sechura, al noroeste del Perú \\
\hline & limatus & Costa árida y vertiente pacífica de Los Andes en el sur de Perú \\
\hline & magister & Andes del sur del Perú y norte de Chile desde el nivel del mar \\
\hline & osgoodi & Altiplano de la Provincia de Arica \\
\hline & osilae & $\begin{array}{l}\text { Sureste del Perú al norte de Argentina. Chuquisaca, Cochabamba, La } \\
\text { Paz, Potosí, Tarija }\end{array}$ \\
\hline & wolffsohni & Oeste de Bolivia \\
\hline & xanthopygus & Chuquisaca, Cochabamba, La Paz, Oruro, Tarija, Potosí \\
\hline \multirow[t]{4}{*}{ Abrocoma } & bennetti & Chile desde Copiapó hasta Bío Bío \\
\hline & boliviensis & Vecindad de Comarapa, Bolivia \\
\hline & cinerea & $\begin{array}{l}\text { Suroeste del Perú, norte de Chile, vertiente oriental de Los Andes en } \\
\text { Argentina. Altiplano Boliviano }\end{array}$ \\
\hline & vaccarum & Provincia de Mendoza \\
\hline \multirow[t]{3}{*}{ Lagidium } & peruanun & Andes centrales del centro y sur del Perú. Parinacota \\
\hline & viscacia & $\begin{array}{l}\text { Sur del Perú, a lo largo de Los Andes hasta el centro-sur de Chile y } \\
\text { Argentina. Cochabamba, La Paz, Oruro, Chuquisaca, Tarija, Potosí }\end{array}$ \\
\hline & wolffshoni & Sur de Chile, sureste de Argentina \\
\hline \multirow[t]{3}{*}{ Microcavia } & australis & $\begin{array}{l}\text { Región montana de Bolivia, sureste del Perú y Chile. Patagonia Argen- } \\
\text { tina y sur de Chile }\end{array}$ \\
\hline & niata & Altiplano del suroeste de Bolivia y norte de Chile, sureste del Perú \\
\hline & shiptoni & $\begin{array}{l}\text { Sólo en las montañas de Tucumán, Catamarca y Provincia de Salta en } \\
\text { Argentina }\end{array}$ \\
\hline Octodontomys & gliroides & $\begin{array}{l}\text { Andes del suroeste de Bolivia, norte de Chile y noroeste de Argentina. } \\
\text { La Paz, Oruro, Potosí }\end{array}$ \\
\hline Octomys & $\operatorname{mimax}$ & Oeste de la región central de Argentina \\
\hline
\end{tabular}


vado en el hemisferio Norte, en Sudamérica se tiene escaso conocimiento de los depósitos cristalizados, los agentes responsables de dichas acumulaciones, así como los sesgos incorporados a las paleomadrigueras. Estos sesgos deben ser considerados al momento de realizar análisis paleoecológicos, y su detección se basa en el conocimiento de la biología e historia natural de los agentes depositadores. En base a la escasa evidencia existente, así como a observaciones hechas en terreno, se presume que especies del género Phyllotis, Abrocoma, Lagidium, Microcavia, Octodontomys y Octomys tendrían la capacidad de generar letrinas cristalizadas en las zonas áridas del sur de Sudamérica (véase Tabla 1, Bozinovic \& Contreras 1990, Pearson \& Christie 1993, Fernández 1994, Fernández \& Panarello 1994, Braun \& Mares 1996, Steppan 1998). La biología de estas especies en general es poco conocida, por lo que la participación de cada una de ellas en la formación de paleomadrigueras debería ser considerada en forma independiente. Asimismo, estudios específicos referentes a hábitos tróficos por ejemplo, deberían realizarse a nivel local, en las cercanías de paleomadrigueras. Ello con el fin de detectar posibles sesgos en la incorporación del material vegetal y animal a cada depósito.

El número de géneros y especies que participan en la formación de paleomadrigueras podría aumentar, luego que se conozca con más detalle la biología e historia natural de las especies de roe-

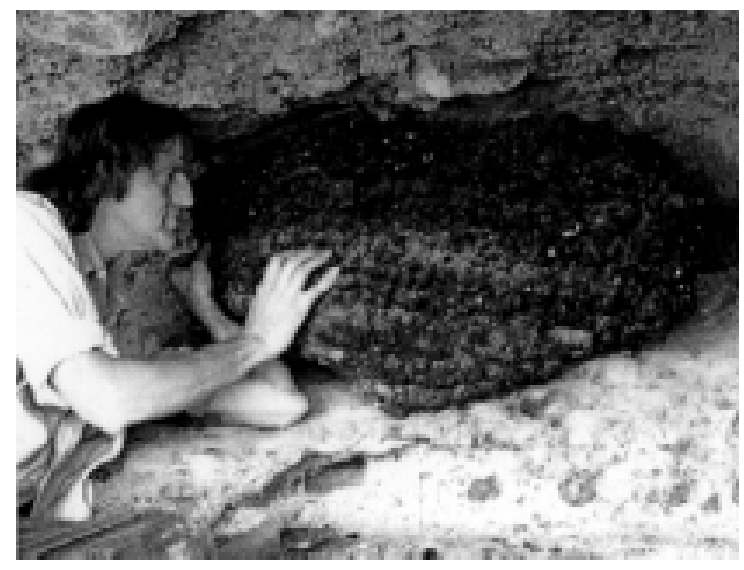

Fig. 2: Paleomadriguera hecha por Neotoma en el desierto de Mojave, al sur del estado de Nevada, Estados Unidos. Este depósito fue fechado en 12.000 años AP. Se muestra al paleoecólogo Paul Martin como escala.

Packrat (Neotoma) midden in the Mojave Desert, south of Nevada State, U.S.A. This midden has been dated in 12,000 radiocarbon years BP. Paleoecologist Paul Martin is shown as size reference. dores que habitan las zonas áridas del sur de Sudamérica. Considerando sin embargo, aquellos elementos comunes a los grupos para los cuales se supone participan en la formación de dichas acumulaciones, junto con la evidencia existente para especies del género Neotoma en Norteamérica, es posible proponer que especies herbívoras, que presenten orina concentrada, de preferencia con hábitos gregarios y que tengan afinidad con ambientes rocosos, podrían ser capaces de generar paleomadrigueras. Estos atributos, en conjunto con la asociación a ambientes con escasa humedad, permiten suponer que especies como Cavia tschudii, Chinchilla brevicaudata, Ch. lanigera, Tympanoctomys barrerae, o especies del género Auliscomys pudieran constituir asimismo agentes depositadores de paleomadrigueras.

\section{MÉTODOS PARA LA DETECCIÓN Y PROCESAMIENTO DE PALEOMADRIGUERAS}

La búsqueda de paleomadrigueras debe centrarse preferentemente en hábitats que contengan formaciones rocosas. Ello se debe a que el establecimiento de paleomadrigueras se ve favorecido en grietas, cuevas o aleros rocosos, formaciones comunes en hábitats de este tipo (Davis 1990). Estas cavidades son utilizadas por los roedores como refugio y sitios de defecación. Las paleomadrigueras de roedores son masas informes, de color gris a café oscuro, fácilmente diferenciables en un sustrato rocoso o el interior de una cueva o alero (Spaulding et al. 1990, Fig. 2). Una vez localizado el depósito es necesario marcar con placa metálica el sitio, y georeferenciar su posición (i.e., latitud y altitud, exposición). Además, se debe describir el tipo de roca en el que se encontró el depósito, su pendiente, así como la vegetación circundante aproximadamente $100 \mathrm{~m}$ al sitio. Luego de ello, el depósito puede ser extraído con un martillo y un cincel. Una vez retirado, debe ser limpiado superficialmente, con el fin de retirar material depositado recientemente. Cada muestra debe ser guardada en una bolsa plástica resistente, y sellada con cinta gruesa para evitar la desintegración posterior del material. En caso de necesidad, y cuando sea posible, la extracción del depósito debe hacerse respetando la estratigrafía natural del mismo. Sin embargo, experiencia acumulada recientemente en Sudamérica indica que las paleomadrigueras se forman rápidamente, en períodos de decenas de años a siglos, por lo cual regularmente no presentarían estratigrafía compleja. Más aún, fechados radiocarbónicos de diferentes estratos de paleomadrigueras, han resultado ser prácticamente idénticos. 
Muchas de las decisiones que se toman durante la recolección de las paleomadrigueras (e.g., tamaño de la muestra, intensidad de limpieza superficial, estratigrafía) son subjetivas, y dependen fundamentalmente de la experiencia de cada investigador (a). Sin embargo, se sugiere extremar las precauciones en el proceso de recolección de los depósitos en terreno, con el fin de evitar la contaminación con material más joven. La contaminación se produce cuando unidades estratigráficas diferentes se mezclan o cuando depositaciones posteriores no son removidas adecuadamente. Ella puede deberse además, a procesos físicos como rehidratación y cristalización de la orina del agente depositador, los cuales pueden introducir sedimento joven a un depósito de mayor edad. Procesos de este tipo se ven favorecidos en cuevas y aleros, dada su naturaleza dinámica, los cuales presentan actividad significativa de aves, insectos y mamíferos, incluyendo al ser humano.

Una vez en el laboratorio, cada muestra debe ser desenvuelta, y se debe descartar del análisis el material disgregado, así como las capas más externas del depósito, con el fin de reducir la probabilidad de contaminación. En el caso que sea posible detectar nuevas unidades estratigráficas, ellas deben ser extraídas en forma separada. Dada la unicidad del depósito es altamente recomendable tomar una submuestra de cada uno (ca. $25 \mathrm{~cm}^{3}$ ó 10-20 g), la cual pueda ser sometida posteriormente a análisis de polen. El resto del depósito debe ser pesado y sometido al proceso de disgregación del material. Para ello, cada muestra debe ser remojada en agua en contenedores individuales por un período de una a dos semanas. Ello permite la disolución de la orina, liberando todo el material animal y vegetal contenido de cada depósito. El tratamiento del material con otros solventes como $\mathrm{HCl}$, no es en absoluto recomendable, por cuanto provoca la denaturación de los ácidos desoxirribonucleicos (ADN) de plantas y animales contenidos en cada muestra. Igualmente, el tratamiento con solventes ácidos destruye la epidermis o capas lipídicas de la materia vegetal contenida en el depósito.

Luego de la disgregación del material, éste debe ser tamizado en filtros de calado conocido (1-2 mm), y lavado con agua corriente hasta eli-

\footnotetext{
${ }^{1}$ National Science Foundation e Inter-American Institute subsidian la datación con espectrofotometría de masas sobre materiales de origen terrestre a investigadores del Cuaternario en Sudamérica. http://www.Colorado.EDU/ INSTAAR/RadiocarbonDatingLab/brochure.html
}

minar todo resto de sedimento y orina. Luego de ello, el material recuperado debe depositarse en un contenedor de papel y secarse en una estufa a $70{ }^{\circ} \mathrm{C}$ por aproximadamente $40 \mathrm{~h}$. Una vez seco, el material debe ser guardado en bolsas individuales con la debida identificación, hasta el momento de su posterior procesamiento.

Con el fin de datar los depósitos recuperados, es necesario tomar una muestra de las fecas contenidas en cada uno de ellos. Esta etapa del proceso se realiza luego del lavado y secado del depósito. El peso de dicha muestra debiera alcanzar al menos $\operatorname{los} 3 \mathrm{~g}$, e idealmente $10 \mathrm{~g}$ para acceder a los métodos regulares de datación radiocarbónica. En caso de no contar con suficiente material fecal para datar el depósito, o algún estrato del mismo, es posible datar pequeños fragmentos vegetales u óseos, utilizando para ello espectrometría de aceleración de masas (Van Devender et al. 1985) ${ }^{(1)}$. Luego de este proceso, el material contenido en el depósito cristalizado puede ser sometido a numerosos análisis, los cuales utilizan como sustrato los restos de macro y micro flora, huesos, insectos, $\mathrm{u}$ otros restos bióticos contenidos en cada depósito.

Como es esperable del decaimiento exponencial con el tiempo de cualquier registro fósil, las paleomadrigueras del Holoceno Tardío son más fáciles de encontrar que registros Pleistocénicos. Parte significativa del trabajo de campo, consiste en predecir dónde se habría optimizado la preservación de las paleomadrigueras. Debido a que la orina cristalizada es soluble en agua, los mejores sitios deberían ser aquellos que han permanecido más secos. Igualmente, una alta humedad relativa durante la estación de verano puede impedir la cristalización de la orina, lo cual explica en parte por qué la preservación de paleomadrigueras decrece desde el suroeste de los Estados Unidos hacia el norte de México, en sitios que reciben el mismo promedio anual de precipitación, pero una mayor proporción del agua cae en verano. Finalmente, la mejor preservación tiende a ocurrir donde la erosión de las formación rocosa es mínima (tipos de roca duro y masivos como piedra caliza, cuarcitas), donde la fisiografía facilite la mantención de la aridez (cuencas pequeñas que faciliten la sequedad de cuevas, aleros y cavidades), y donde la precipitación promedio sea menor que $300 \mathrm{~mm}$ al año. Ello no significa que las paleomadrigueras Pleistocénicas se encuentren exclusivamente en estas situaciones. En los desiertos más secos tanto de Norteamérica (Mojave, Vizcaino) como de Sudamérica (Atacama), hemos encontrado paleomadrigueras Holocénicas y Pleistocénicas bajo grandes cantos rodados, obviamente una situación no ideal para la preservación de este regis- 


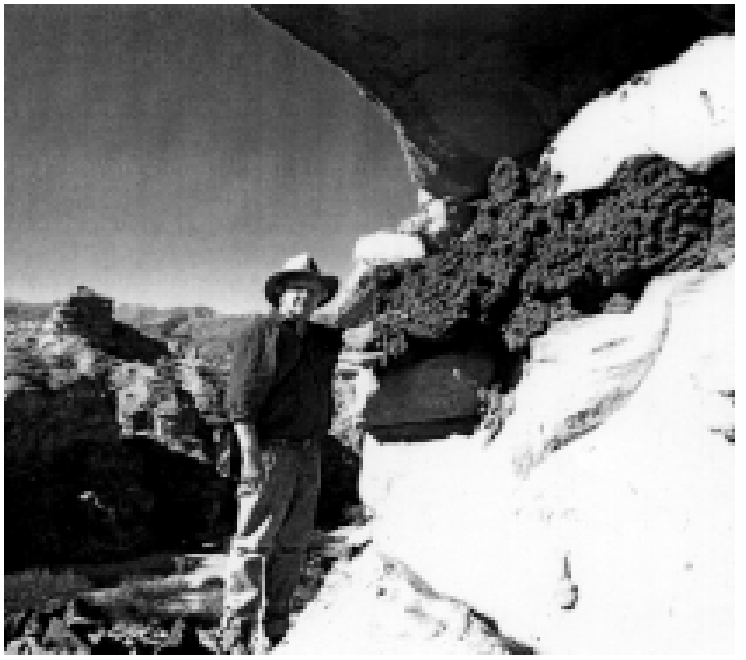

Fig. 3: Paleomadriguera hecha por Lagidium en alero de arenisca en Talampaya, Provincia de La Rioja, Argentina. Diferentes niveles de este depósito fueron fechados en $1.075 \pm 85,3.860 \pm$ 85 , y $4.025 \pm 105$ años AP. Se muestra el geólogo Wolf Volkheimer como escala.

Viscacha (Lagidium) midden found in a sandy rockshelter in Talampaya, Provincia de La Rioja, Argentina. Different levels of this deposit have been dated in $1,075 \pm 85,3,860$ \pm 85 , and 4,025 \pm 105 radiocarbon years BP. Geologist Wolf Volkheimer is shown as size reference.

tro en zonas menos áridas. Existe una conexión fuerte entre la erosión y la preservación de las paleomadrigueras. Este patrón ha permitido por ejemplo estimar la tasa de erosión en el Gran Cañón del Colorado, utilizando para ello la edad de las paleomadrigueras y la distancia a la pared de la formación rocosa (Cole \& Meyer 1982). En tipos de roca donde la erosión es rápida, las paleomadrigueras comúnmente están expuestas o "colgando" de los roqueríos (Fig. 3).

\section{CONSIDERACIONES TAFONÓMICAS}

Muchas de las especies de roedores que generan paleomadrigueras tienen simpatría y sobreposición geográfica. Ello determina que algunas zonas presenten paleomadrigueras generadas por diferentes agentes depositadores. Dado que las preferencias tróficas de cada especie de roedor pueden introducir sesgos específicos al depósito cristalizado, es necesario discriminar entre agentes depositadores (e.g., Betancourt et al. 1986). Para ello existen diversas posibilidades. En primer lugar es posible utilizar la forma y tamaño de las fecas que componen el registro. Según esto, sería posible diferenciar inicialmente entre roe- dores Múridos o de tamaño pequeño, de roedores Histricognatos, o tamaño más grande. La discriminación entre agentes depositadores dentro de cada grupo requiere del conocimiento acabado de la forma y tamaño de las fecas que cada especie presenta en la actualidad. Ello precisa de la realización de observaciones específicas destinadas a caracterizar tanto los valores promedio de las fecas de cada taxón, así como su varianza. Esta variabilidad debería reflejar diferencias tróficas, geográficas, o de tamaño, así como otras variables de interés.

Otras técnicas más sofisticadas pueden ser utilizadas cuando el análisis de fecas no resulta concluyente. En primer lugar, es posible desarrollar análisis morfológico de pelos contenidos en las paleomadrigueras. Ellos contemplan desde descripciones de morfología hechas bajo lupa estereoscópica, hasta observaciones hechas con microscopía electrónica (e.g., Piantanida \& Petriella 1976, Aravena et al. 1989). Este proceso requiere sin embargo, la información que describa los atributos específicos de cada tipo de pelo, así como la variabilidad que éste puede presentar. En segundo lugar, es posible realizar inmunoensayos con la albúmina contenida en la orina de cada depósito cristalizado (Lowenstein et al. 1991). Ello permite el reconocimiento de proteínas específicas, las cuales normalmente se conservan en buen estado dada la naturaleza del depósito fecal. La aplicación de esta técnica requiere sin embargo, del conocimiento previo de las proteínas contenidas en la orina de cada especie, la composición química y mineralógica de la misma, así como de los anticuerpos específicos necesarios para la detección. Por último, sería posible adscribir el agente depositador de la paleomadriguera utilizando análisis de ADN. Ello podría realizarse sobre células epiteliales contenidas en la orina del depósito, las cuales pueden ser sometidas a extracción y amplificación de ADN utilizando técnicas de PCR (Rogers \& Bendich 1985). Trabajos de esta naturaleza han permitido asignar a Nothrotheriops shastensis (Perezoso) en fecas contenidas en depósitos Pleistocénicos del desierto de Nevada (Poinar et al. 1998). Recientemente, M.N.R. Kuch, J.L. Betancourt, C. Latorre, S. Steppan \& H.N. Poinar (resultados no publicados) analizaron el ADN mitocondrial obtenido desde fecas, pudiendo identificar a Phyllotis limatus como el agente formador de una paleomadriguera de 11.700 años colectada en el eje sur del Salar de Atacama, en el norte de Chile. En la actualidad, $P$. limatus no habita las cercanías de la paleomadriguera, encontrándose $100 \mathrm{~km}$ hacia el norte, lo que indica un cambio en su rango de distribución. 
El análisis de los sesgos contenidos en las paleomadrigueras de roedores requiere del conocimiento de las preferencias tróficas de cada agente depositador. Esta información permite discriminar patrones en dinámica vegetacional, de variaciones en la dieta de los agentes responsables de la depositación. Información de este tipo sólo puede provenir de observaciones actuales realizadas en roedores nativos distribuidos en sus hábitats naturales, no pudiendo ser extrapolada a partir de trabajos anteriores realizados en otras latitudes y con otras especies.

Finalmente, cuando se trabaja con paleomadrigueras, es necesario considerar que la muestra de vegetación contenida en cada depósito está sesgada respecto de la vegetación aledaña de la zona, encontrándose sobrerepresentados aquellos hábitats asociados a laderas rocosas. Dado que los depósitos fósiles sólo se conservan en aquellas zonas rocosas distribuidas en hábitats de escasa humedad, zonas de escasa pendiente, alejadas de las áreas de madrigueras de los roedores, quedarían subrepresentadas en paleoreconstrucciones vegetales basadas en paleomadrigueras.

\section{USO ACTUAL Y POTENCIAL DE PALEOMADRIGUERAS}

La evidencia contenida en las paleomadrigueras permite abordar preguntas en diversas áreas del conocimiento como son botánica, zoología, ecología, biogeografía, arqueología, climatología, entre otras. Con el fin de ilustrar la potencialidad de dichos registros, se describen a continuación algunos ejemplos en los que se ha utilizado paleomadrigueras de roedores como fuente primaria de información.

En primer lugar, el análisis de paleomadrigueras de roedores ha sido utilizado directamente para reconstruir la vegetación en zonas áridas y semiáridas de Norteamérica representando 35 años de estudios regionales realizados por una docena de investigadores (e.g., Wells \& Jorgensen 1964, Wells 1966, 1970, 1976, 1983, Wells \& Berger 1967, Mehringer \& Ferguson 1969, Van Devender \& King 1971, Lanner \& Van Devender 1974, Phillips \& Van Devender 1974, Leskinen 1975, King 1976, Van Devender \& Mead 1976, Spaulding 1977, 1983, 1985, Van Devender 1977, 1987, Van Devender \& Everitt 1977, Van Devender et al. 1978, 1984, 1990, 1991, 1994, Thompson 1979, Van Devender \& Riskind 1979, Van Devender \& Spaulding 1979, Betancourt \& Van Devender 1981, Lanner \& Van Devender 1981, Mead \& Phillips 1981, Cole 1982, 1983, 1985, 1986, 1990, Thompson \& Mead 1982, Mead et al. 1983, Betancourt 1984, Betancourt \& Davis
1984, Van Devender \& Burgess 1985, Thompson 1985, Cole \& Webb 1985, Wells \& Woodcock 1985, Spaulding \& Graumlich 1986, Woodcock 1986, Cinnamon \& Hevly 1988, Anderson \& Van Devender 1991, Betancourt et al. 1991, 2000b, Thompson 1992, Van Devender \& Wiens 1993, Jennings \& Elliott-Fisk 1993, Hunter \& McAuliffe 1994, Nowak et al. 1994, Koehler \& Anderson 1994, Van Devender \& Hall 1994, Rhinehart \& McFarlane 1995, Koehler \& Anderson 1995, Rhode \& Madsen 1995, Anderson \& Van Devender 1995, McAuliffe \& Van Devender 1998, Lanner \& Van Devender 1998, Hunter et al. 2001, ver síntesis Spaulding et al. 1983, Van Devender et al. 1987, Betancourt et al. 1990a, Thompson et al. 1993). La gran cantidad de paleomadrigueras datadas en la zona (ca. 2.500), las cuales abarcan un período de tiempo de ca. de 40.000 años, ha permitido además calibrar con precisión los eventos asociados a cada momento de cambio climático. Con ello se ha podido localizar los movimientos de vegetación asociados a glaciaciones Pleistocénicas, los cuales han mostrado la existencia de comunidades más dinámicas de lo que inicialmente era pensado a partir de estudios biogeográficos (Betancourt et al. 1990b).

Una historia de la vegetación en los desiertos Sudamericanos, comparable a la conocida para Norteamérica, puede ayudar a resolver preguntas clásicas relacionadas con el paleoclima, como la precisa extensión de los vientos alisios durante el último período glacial, o si el Younger Dryas influenció el clima sudamericano (Heusser 1993, Markgraf 1993, Moreno 1997). Por ejemplo, en el Altiplano de Chile, sobre San Pedro de Atacama, incrementos en el máximo nivel lacustre durante el Pleistoceno Tardío y el Holoceno Temprano, han sido atribuidas a la intensificación de los Alisios del Noreste y a un "invierno boliviano" más intenso (Grosjean 1994, Grosjean et al. 1995, Valero-Garcés et al. 1996). Ello habría incrementado la llegada de humedad hacia el oeste de Los Andes, definiendo el cinturón de lluvia tropical en verano, siendo las fuentes de humedad dominantes a través de los Andes centrales. Sin embargo, hoy en día se sabe que las tormentas de invierno contribuyen hasta un $50 \%$ de la precipitación anual en el norte hasta los $24^{\circ}$ $S$ en el desierto de Atacama, fundamentalmente como incursiones de sistemas frontales fríos y celdas de baja presión (Vuille \& Ammain 1997). La expansión hacia el norte de los alisios del oeste durante el período glacial tardío, ha sido utilizada para explicar las condiciones de mayor humedad de Chile central durante este período (Villagrán \& Varela 1990). Variaciones en el nivel lacustre en el altiplano chileno pueden de- 
berse igualmente a un incremento en las precipitaciones de invierno, más que las precipitaciones estivales. Paleomadrigueras localizadas en toda la extensión del desierto de Atacama (16-28 ${ }^{\circ} \mathrm{S}$ ) podrían ser utilizadas para circunscribir las influencias geográficas de la lluvia y de cinturones de lluvia extra tropicales durante los últimos 40.000 años. Asimismo, esta información podría ser utilizada en comparaciones interhemisféricas acerca de la naturaleza y temporalidad de los cambios climáticos en el continente Americano (Markgraf 1998). Particularmente relevante podría resultar la sincronía en cambio climático en el desierto de Atacama y sus regiones adyacentes, las cuales puedan generar hipótesis relacionadas con las fuerzas orbitales y la distribución latitudinal y estacional de la insolación (Berger \& Loutre 1991), así como teleconexiones climáticas asociadas con la formación de aguas profundas del Atlántico Norte (Broecker 1998). Un transecto de paleomadrigueras de roedores a través del desierto de Atacama podría ser utilizado para poner a prueba hipótesis biogeográficas tales como las propuestas por Villagrán et al. (1983), Arroyo et al. (1988), o Moreno et al. (1994), las que explican los patrones actuales de distribución de la biota en base a la existencia de corredores (o barreras) vegetacionales dinámicos, los que se habrían producido debido a cambios climáticos ocurridos durante el Cuaternario en el norte de Chile. Redes de paleomadrigueras pueden ser utilizadas para poner a prueba hipótesis relacionadas con bosques relictos en las montañas del Noroeste argentino (Nores \& Cerana 1990), o con el grado de endemismo de la flora del sur de Sudamérica (Crisci et al. 1991, Roig 1994).

Las implicancias que puede tener el análisis de paleomadrigueras de roedores no se limitan a procesos bióticos. El análisis de dichos depósitos ha permitido abordar preguntas relacionadas con la actividad magnética de la atmósfera terrestre. Ello gracias al efecto que la radiación cósmica ejerce sobre la formación de átomos específicos (e.g., ${ }^{14} \mathrm{C},{ }^{36} \mathrm{Cl},{ }^{10} \mathrm{Be},{ }^{26} \mathrm{Al},{ }^{3} \mathrm{He}$ ). Estos isótopos son utilizados en datación geológica, análisis geomorfológico, hidrológicos, así como marcadores geológicos (Plummer et al. 1997). La tasa de formación de dichos isótopos varía en el tiempo y en el espacio, dependiendo de las condiciones de actividad magnética de la Tierra, así como de las condiciones locales de depositación. El estudio de los cambios en la razón ${ }^{36} \mathrm{Cl} / \mathrm{Cl}$ realizado a partir de los isótopos contenidos en la orina cristalizada de Neotoma durante los últimos 40.000 años, ha permitido mostrar la gran variabilidad asociada a la producción de dicho isótopo (Plummer et al. 1997). Esta variabilidad se expli- caría por cambios en las condiciones climáticas asociadas a las glaciaciones del Cuaternario, las cuales habrían afectado los procesos de aridificación y desecación de lagos pluviales. Procesos como éste podrían haber sido de gran importancia en las zonas áridas del Norte de Chile, donde se reconoce la existencia de cambios en la dinámica de las aguas límnicas de la zona. Explicaciones alternativas, incorporan el movimiento de la corriente del chorro ("jet stream") asociado a las mencionadas glaciaciones del Cuaternario (Plummer et al. 1997).

Registros de paleotemperatura y humedad ambiental han sido obtenidos analizando el contenido isotópico (hidrógeno, oxígeno y carbono) en la celulosa de las plantas encontradas en paleomadrigueras de Neotoma (Long et al. 1990, Pendall et al. 1999), permitiendo la reconstrucción paleoclimática de numerosas áreas de zonas áridas en el noroeste de Norteamérica (Betancourt et al. 1990b). La información contenida en paleomadrigueras de roedores constituye una evidencia independiente de los cambios climáticos que han operado durante el Cuaternario. Ella puede ser usada como elemento de validación de modelos paleoclimáticos desarrollados en base a evidencia proveniente de hielos glaciares, sedimentación u otros (e.g., Bartlein et al. 1998), lo que permitiría comprender y mejorar los modelos climáticos globales existentes.

Las paleomadrigueras de roedores no sólo contienen restos de la vegetación circundante a las madrigueras, sino que además pueden contener restos de fauna de vertebrados (e.g., roedores, lagomorfos, quirópteros, Van Devender \& Bradley 1990), así como de invertebrados (e.g., insectos, Hall et al. 1990, Elias \& Van Devender 1990, 1992, Elias 1992, Elias et al. 1995, Van Devender \& Hall 1994). Análisis de paleomadrigueras han sido de gran utilidad para conocer la dinámica Cuaternaria de la entomofauna que habita zonas áridas de Norteamérica, encontrándose que ésta ha permanecido escasamente alterada durante los últimos eventos climáticos ocurridos durante el Cuaternario (e.g., Hall et al. 1990). La fauna de vertebrados por el contrario, habría presentado mayor variabilidad durante este mismo período.

El estudio de paleomadrigueras ha servido para evaluar patrones biogeográficos de distribución de especies, como el observado para Larrea tridentata en los desiertos del oeste norteamericano (Hunter et al. 2001). Allí, la jarrilla presenta un patrón de distribución desde el sureste al noroeste, presentando razas con mayor ploidía, asociadas a zonas de mayor aridez y temperatura. De hecho, se propuso que Larrea habría desarrollado poliploidía a medida que la especie migró progre- 
sivamente a áreas que presentaban veranos más secos y cálidos, a medida que migraba desde áreas más húmedas de México, hacia zonas de mayor latitud del suroeste de los Estados Unidos. Estos cambios habrían ocurrido luego de las glaciaciones Pleistocénicas, cuando se incrementó la aridez a comienzos del Holoceno. Sin embargo, análisis morfológicos de restos de Larrea recuperados en numerosas paleomadrigueras del oeste de Norteamérica, han permitido establecer que las razas di y tetraploides de Larrea habitaban la zona durante el Último Máximo Glacial, y que la actual distribución de ploidía se habría originado en diversos eventos vicariantes y de dispersión, no estando asociado directamente con la colonización de hábitats áridos (Hunter et al. 2001).

El registro vegetal de larga data contenido en los depósitos cristalizados de roedores ha permitido además, evaluar la dinámica en patrones filéticos de especies vegetales. Es así por ejemplo, que especies del genero Sophora, evidencian una gran estabilidad filética. La comparación de morfología externa macro y microscópica de diferentes especímenes actuales y fósiles del género, en conjunto con especímenes procedentes de diversos puntos de distribución actual de este género, ha permitido comprobar la constancia morfológica y anatómica entre los taxa examinados (Northington et al. 1977). Este patrón sugiere una disyunción reciente de las poblaciones a partir de un antepasado común (Northington et al. 1977).

El uso de paleomadrigueras de roedores ha resultado adecuado además, para el análisis de patrones microevolutivos de roedores (e.g., Smith et al. 1995, Smith \& Betancourt 1998). Smith et al. (1995) muestran cambios en el tamaño corporal de Neotoma cinerea en respuesta a variaciones en temperatura ocurridas durante los últimos 25.000 años. Estos roedores habrían seguido la regla de Bergmann, presentando tamaño corporal más grande en los períodos de menor temperatura registrados durante el Cuaternario en el suroeste de Norteamérica. Dado que en las zonas áridas de Sudamérica, han existido cambios climáticos significativos durante el Cuaternario (Arroyo et al. 1988), el análisis de paleomadrigueras de roedores sudamericanos, podría permitir develar la plasticidad de la fauna nativa a dichos cambios. Asimismo, dada la gran cantidad de zonas áridas que presentan paleomadrigueras producidas por diferentes roedores (véase Betancourt et al. 1990b), así como los numerosos cambios climáticos a que dichas zonas han estado sometidas durante el Cuaternario, en teoría sería posible incorporar la dimensión temporal en estudios macroecológicos. Ello por cuanto sería posible comparar patrones de tamaño, peso, u otra variable relevante entre diferentes zonas áridas del mundo, permitiendo evaluar la generalidad de los patrones encontrados, ya no sólo en el espacio, sino además en el tiempo.

El análisis de macrorestos vegetales contenidos en paleomadrigueras de roedores durante el Cuaternario ha permitido establecer las respuestas fisiológicas de plantas a los cambios en $\mathrm{CO}_{2}$, temperatura y humedad a través del análisis de densidad estomatal y razón de ${ }^{13} \mathrm{C} /{ }^{12} \mathrm{C}$ ó $\delta^{13} \mathrm{C}$ (Van de Water et al. 1994). Van de Water et al. (1994) mostraron una disminución en la densidad estomatal en agujas de Pinus flexilis, y una disminución en el contenido de ${ }^{13} \mathrm{C}$ de las mismas. Ello como respuesta de las plantas al incremento en el contenido de $\mathrm{CO}_{2}$ atmosférico asociado al proceso de deglaciación. Estudios de este tipo, establecen un nexo explícito entre trabajos realizados a escalas de tiempo y espacio disímiles, como son los estudios de ecofisiología vegetal y estudios de cambio climático a gran escala durante el Cuaternario. Trabajos como este cobran gran relevancia en la actualidad, debido al proceso de cambio global que estaría experimentado actualmente nuestro planeta.

La evidencia contenida en las depositaciones cristalizadas de roedores ha permitido conocer el origen y dinámica de colonización de poblaciones marginales de Pinus edulis, en el suroeste de Norteamérica (Betancourt et al. 1991). Macrorestos y polen de paleomadrigueras de Neotoma con un rango de edad de 5.000 años, en conjunto con información dendrocronológica procedente de individuos del mismo sitio, han permitido datar el inicio de la colonización de este árbol entre 1.290-420 años AP. La evidencia fósil, en conjunto con información genética obtenida a partir de ensayos electroforéticos de individuos que actualmente componen dichos bosques, ha permitido inferir además los eventos fundadores que habrían originado dichas poblaciones (Betancourt et al. 1991). Trabajos como éste demuestran que el uso de evidencia paleoecológica en conjunto con evidencia neontológica, aportan elementos poderosos para comprender más acabadamente la biología de poblaciones marginales (Betancourt et al. 1991).

Asimismo, el análisis de paleomadrigueras de roedores ha sido utilizado con éxito en el estudio del efecto que poblaciones humanas prehistóricas ejercieron sobre su medio ambiente (Betancourt \& Van Devender 1981, Fall 1990). Un ejemplo dramático se observa en poblaciones de Anazasi, habitantes de zonas áridas del suroeste de Norteamérica, los cuales habrían agotado sus recursos forestales y con ello probablemente deter- 
minado su propia desaparición (Betancourt \& Van Devender 1981). En zonas áridas como el Desierto de Atacama, las cuales por al menos 11.000 años han sustentado población humana importante, el estudio de paleomadrigueras de roedores podría resultar crucial para entender procesos de poblamiento y abandono de dichas áreas. Esta evidencia permitiría conocer asimismo los patrones de clima y vegetación que sustentaron la primera colonización de la zona de Atacama durante en Holoceno temprano (11.0009.000 años AP) (Grosjean 1994, Keefer et al. 1998, Sandweiss et al. 1998), o durante el Holoceno medio, en el llamado período del "Silencio Arqueológico" (Núñez 1983, 1994). En el altiplano boliviano, las paleomadrigueras pueden entregar información paleoambiental adicional, que permita poner a prueba hipótesis específicas sobre la emergencia y colapso de la cultura Tiwanaku (Binford et al. 1997). Además, el estudio de paleomadrigueras aledañas a asentamientos indígenas favorecería el análisis de procesos de domesticación de especies vegetales nativas de la zona.

Las paleomadrigueras pueden ser utilizadas además para determinar la línea de referencia y condiciones ambientales basales previas al establecimiento de colonizadores Europeos en América. Ello puede ser útil para la discriminación entre factores causales de los cambios ambientales que han ocurrido en el siglo XX. Es así por ejemplo, que las paleomadrigueras han sido usadas para discriminar el origen de algunas plantas. Tal es el caso de Corispermum (Quenopodiaceae), la cual supuestamente habría sido introducida desde Eurasia en el Siglo XIX. Ella sin embargo, está presente en todo el Oeste Norteamericano desde al menos los últimos 30.000 años (Betancourt et al. 1984). En la misma área, se ha propuesto que la expansión de algunas especies leñosas nativas se debería a la supresión de fuego y pastoreo. Sin embargo, el análisis de paleomadrigueras muestra cómo la dinámica poblacional en el límite de distribución Holocénica reciente puede ser alterado por efectos antropogénicos (Swetnam et al. 1999). Asimismo, esta evidencia puede contribuir a evaluar el efecto del cambio de régimen de fuego en invasiones reciente de árboles sobre praderas a lo largo del límite bosque-estepa en la Patagonia (Markgraf et al. 1997), así como la aparente invasión de arbustos en el desierto del Monte, en Argentina. La suspensión del fuego en la Patagonia puede estar empujando los árboles hacia la pradera, mientras que el fuego de origen antrópico en el Monte, puede estar aumentando la biomasa de algunas especies leñosas, a expensa de otras. Este hecho determinó la desaparición de Schinus polygamus en el este de la precordillera cercana a Mendoza (Roig \& Ambrossetti 1971) favoreciendo praderas dominadas por Pappophorum caespitosum y Stipa gyneriodes (Martínez 1984). La quema permanente que se hace para mantener estas praderas ha generado pendientes desnudas, debido a la pérdida de suelo y a la volatilización del nitrógeno, generando una disminución sustancial de biodiversidad. La quema sucesiva realizada en la Patagonia, ha promovido la expansión de Colliguaya integerrima, un arbusto nativo que se reproduce asexualmente, tóxico para el ganado. En muchas áreas, $C$. integerrima forma parches monoespecíficos, de bajo valor para la conservación. Las paleomadrigueras podrían ser utilizadas en casos como este, para documentar las condiciones ambientales basales previas al incremento de los fuegos antrópicos, con el fin de comparar las tasas de cambio del establecimiento de los colonizadores europeos y nativos. Pensamos que esta aproximación comparativa aportaría herramientas útiles a aquellas personas que usan estas tierras, tanto en Norte como en Sudamérica.

\section{EL ESTADO DEL CONOCIMIENTO DE PALEOMADRIGUERAS EN SUDAMÉRICA}

Hasta el momento, gran parte del estudio de paleomadrigueras se ha centrado en la realización de expediciones de reconocimiento en diferentes países, así como en la ejecución de numerosos proyectos laterales que utilizan el rico archivo de restos animales y vegetales identificados en las paleomadrigueras. Dos instituciones con fuerte tradición en estudios paleoecológicos (Laboratorio de Sistemática y Ecología Vegetal, Universidad de Chile, Santiago, Chile y el Instituto Argentino de Nivología y Glaciología, CRICYT, CONICET, en Mendoza, Argentina) han equipado sus dependencias con los elementos básicos para procesar y analizar los depósitos. Se han realizado prospecciones en Argentina, Bolivia y Chile. Hasta el momento se han colectado más de 500 paleomadrigueras, de las cuales una porción significativa ha sido analizada. Como referencia, el trabajo de las últimas cuatro décadas en el análisis de paleomadrigueras en el oeste Norteamericano ha producido más de 2.500 paleomadrigueras datadas.

En Argentina, un total de 185 paleomadrigueras han sido recolectadas desde el límite sur del altiplano andino en Jujuy hasta el norte de la Patagonia en la Provincia de Río Negro. Se ha intentado completar cronologías amplias con las paleomadrigueras, provenientes de una variedad de eleva- 
ciones (660-3.000 m de altitud) y tipos de roca. Por ejemplo, la serie del Cerro Bayo proviene de un batolito de granito, en Talampaya desde un depósito de areniscas fluviales del Triásico, en el Huaco de calizas marinas del Ordovicico, en Sierra Cacheuta desde riolitas, basaltos en la Gruta del Indio, calizas jurásicas en la Cueva de las Brujas y Las Lajas y toba riolítica en el Valle Encantado/La Primavera. Algunos sitios han sido seleccionados por su cercanía al público en general y a científicos en particular, con el fin de hacer más asequible la información de paleomadrigueras. En la Provincia de La Rioja por ejemplo, se está desarrollando una secuencia de 10.000 años basada en paleomadrigueras, en el Parque Provincial de Talampaya, una atracción pública que contiene un espectacular cañón moldeado en areniscas Triásicas de color rojo. En la actualidad, se encuentra en preparación un manuscrito que elabora los cambios climáticos en Sierra Cacheuta durante los últimos 11.000 años. Destaca de este registro, la aparente estabilidad de la vegetación del Monte durante el Holoceno, lo cual podría explicarse por la gran amplitud ecológica de gran parte de los taxa vegetales del Monte. En muchos sitios del Monte argentino, una reducción en el promedio anual de temperatura de $3{ }^{\circ} \mathrm{C}$, el equi-

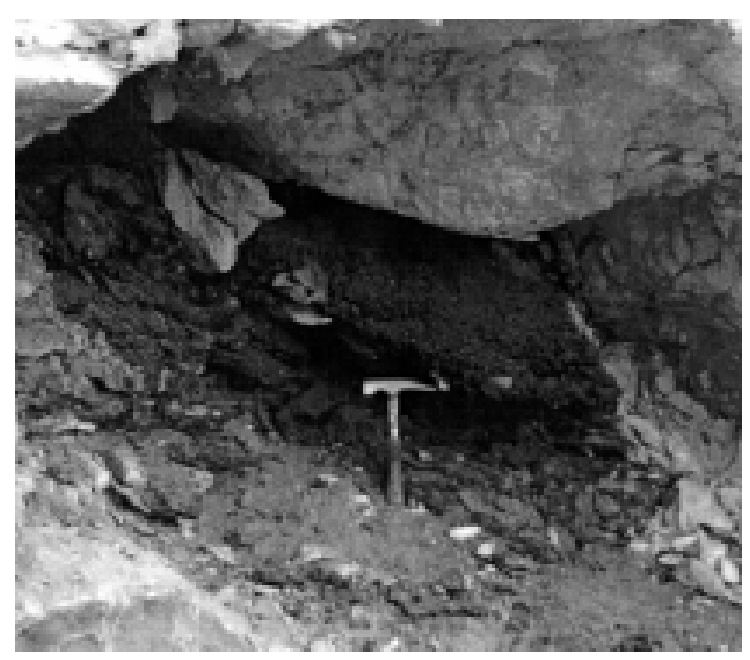

Fig. 4: Paleomadriguera de Lagidium dentro de cueva de caliza en localidad de Las Lajas, Provincia de Neuquén, Argentina. Este depósito también contiene fecas de un perezoso extinto. Las fecas de Lagidium fueron fechadas en $13.750 \pm 230$, y las del perezoso en $13.730 \pm 1.070$ y $14.665 \pm 150$ años AP.

Lagidium midden from a limestone in Las Lajas, Provincia de Neuquén, Argentina. This deposit also contains feces of an extinct sloth. Lagidium feces have been dated in 13,750 \pm 230 radiocarbon years BP, and sloth feces are 13,730 \pm $1,070-14,665 \pm 150$ years old. valente de $500 \mathrm{~m}$ en altitud, produciría más o menos la misma vegetación, y el mismo registro de paleomadrigueras, que hoy. El gradiente de vegetación con altitud es tan gradual que la mayoría del Monte, excepto en las misma márgenes, es un lugar poco sensible a cambios climáticos.

Se está analizando igualmente, una serie de paleomadrigueras provenientes del sitio arqueológico Gruta del Indio, cerca de San Rafael, Mendoza, el cual es conocido por la presencia de megafauna extinta, especialmente el perezoso gigante (D'Antoni 1983, Long et al. 1998). Una de las paleomadrigueras del norte de la Provincia de Neuquén producida por Lagidium, datada en 13.750 años, contiene abundante pelo y fecas de un herbívoro extinto (Fig. 4). Análisis filogenéticos hechos sobre 573 pb de la región $12 \mathrm{~S}$ de ADN mitocondrial, muestran que las fecas fueron hechas por una especie de perezoso para la cual no se cuenta aún con restos óseos, la cual estaría relacionada lejanamente con las cuatro otras especies vivas o extintas de perezoso. Análisis de polen y cutículas de plantas, así como de fragmentos del gen rbcl de cloroplastos de 110 $\mathrm{pb}$, indican que este perezoso ramoneó muchas de las hierbas, pastos y arbustos que aún son comunes en el sitio. Este hábitat correspondería a la estepa arbustiva de la Patagonia (M. Hofreiter, J.L. Betancourt, A. Pellida, X. Sbriller \& H.G. Mcdonald resultados no publicados).

Cerca de Bariloche, se están estudiando paleomadrigueras a lo largo de un transecto EsteOeste de $250 \mathrm{~km}$, que abarca la transición entre el bosque de Nothofagus y la estepa patagónica. Este es uno de los gradientes ambientales más interesante entre las zonas áridas de Sudamérica: a pesar de pequeñas diferencias en elevación $(1.200$ a $900 \mathrm{~m})$, la lluvia decae dramáticamente desde más de 1.000 a $200 \mathrm{~mm}$ en una escasa longitud de $250 \mathrm{~km}$. En particular, interesa conocer la estabilidad a largo plazo del ecotono bosque-estepa, en relación al uso de la tierra, fuego y clima (Markgraf et al. 1997). Particular interés presenta la transición bosque patagónico/estepa cerca del Valle Encantado/La Primavera y Las Lajas, así como el Desierto del Monte cerca de la Gruta del Indio. Si los alisios hubiesen estado desplazados hacia el norte durante el último período glaciar, entonces taxa sureños como Austrocedrus, Araucaria y Nothofagus podrían haberse expandido hacia el norte junto a tormentas y condiciones de temperatura más baja, durante la época de crecimiento. Macrofósiles de estos taxa deberían entonces estar presentes en la superficie de paleomadrigueras Pleistocénicas en estas áreas. Desafortunadamente, hasta ahora sólo se ha encontrado una paleomadriguera con esa 
edad en esta zona transicional (Las Lajas), y la muestra no contenía evidencia de estos árboles.

Contrario al gran avance existente en Argentina, en Bolivia sólo se han realizado prospecciones breves en el campo, con investigadores de la Universidad Mayor de San Andrés (La Paz) y ORSTOM en Julio de 1997. Allí se encontraron paleomadrigueras tan al norte como los $20^{\circ} \mathrm{S}$ y tan altas como a los $3.800 \mathrm{~m}$. Es posible encontrar paleomadrigueras en todo el Altiplano Boliviano, aunque en baja abundancia, siendo posible encontrar sólo unas cuantas paleomadrigueras en cada sitio. Aparentemente, los mejores sitios para la conservación de estos depósitos se encuentran en el desierto, justo en el borde del Altiplano, en la parte más austral del país (cerca de Camargo y el distrito de Tarija).

Para el norte de Chile y sur de Perú, se han desarrollado trabajos que forman parte integral de un transecto de $1.600 \mathrm{~km}$ de estudios paleoambientales en el Desierto de Atacama (16$26^{\circ} \mathrm{S}$ ). Más de 400 paleomadrigueras han sido colectadas en cuatro áreas en el norte (Arequipa, Peru, $16^{\circ} \mathrm{S}, 2.350-2.750 \mathrm{~m}$; Sierra Huaylillas, $18^{\circ} \mathrm{S}, 2.700-3.400 \mathrm{~m}$ ) (Holmgren et al. 2001), centro (cuencas de Calama y San Pedro Atacama, 22-24 $\mathrm{S}, 2.400-3.300 \mathrm{~m}$ ) (Betancourt et al. 2000a, Latorre et al. en prensa, C. Latorre, J.L. Betancourt, K.A. Rylander, J. Quade \& O. Matthei resultados no publicados) y sur de Atacama (Quebrada de Chaco, $\sim 25^{\circ} 30^{\prime}$ S, 2.650-3.500 m). Los datos obtenidos indican que en el pasado la vegetación, incluyendo pastos de estepa, se expandió en lo que hoy día corresponde al desierto absoluto (i.e., sin lluvia o plantas vasculares). En el área cercana a Calama y el Salar de Atacama, esta expansión se debió a importantes incrementos en la lluvia de verano entre los 16.200 y 10.500 años AP (Betancourt et al. 2000a, Latorre et al. 2002, C. Latorre, J.L. Betancourt, K.A. Rylander, J. Quade \& O. Matthei resultados no publicados, Fig. 5). En el área al sur del Salar de Punta Negra y el Parque Nacional Llullaillaco, la entrada de vegetación en el desierto absoluto $(\sim 2.600 \mathrm{~m})$ ocurrió debido a incrementos en la precipitación invernal entre los 23.000-18.000 años AP (J. Betancourt et al. resultados no publicados). Esta historia de la vegetación, obtenida a partir del análisis de paleomadrigueras, está ayudando a entender la historia tanto de los vientos tropicales del este como la de los vientos del oeste en Sudamérica, así como sus efectos en la historia biogeográfica del Desierto de Atacama.

El paleoclima del Cuaternario de los Andes centrales está poco claro, debido a numerosas discrepancias entre diversos registros "proxi" los cuales se han obtenido para esta compleja región, tanto geográfica como climática (véase http:// wwwpaztcn.wr.usgs.gov/pcaw). La edad exacta, duración y magnitud de fases húmedas y secas rara vez son replicadas para algún proxy, y han existido pocas oportunidades para comparar el mismo período de tiempo para un mismo proxy, pero en numerosos sitios o entre regiones. La historia de la vegetación obtenida desde paleomadrigueras provee una oportunidad como ésta para la vertiente pacífica de Los Andes.

\section{CONSIDERACIONES FINALES}

En Norteamérica, los depósitos fecales cristalizados han sido estudiados por 35 años, mientras que
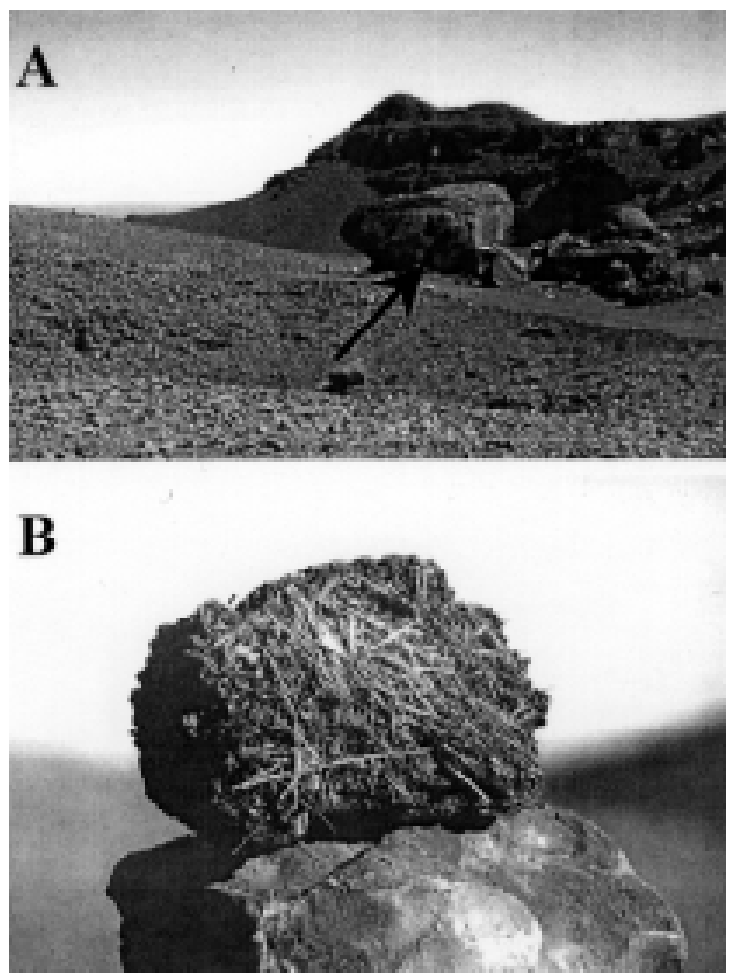

Fig. 5: (A) Alero en Cordón de Tuina cerca de Calama, Segunda Región, Chile con (B)

paleomadriguera fechada en $10.140 \pm 160$ años AP. Este depósito contiene diversos taxa vegetales (e.g., Baccharis tola, B. boliviensis, Anatherostipa venusta) que hoy existen entre $500-800 \mathrm{~m}$ por sobre la localización de la paleomadriguera (C. Latorre, J.L. Betancourt, K.A. Rylander, J. Quade \& O. Matthei resultados no publicados).

(A) Rockshleter in Cordón de Tuina, near Calama, Segunda Región, Chile with (B) midden dated in 10,140 \pm 160 radiocarbon years BP. This deposit holds several plant taxa (e.g., Baccharis tola, B. boliviensis, Anatherostipa venusta) that exist today 500-800 $\mathrm{m}$ above present day midden localization (C. Latorre, J.L. Betancourt, K.A. Rylander, J. Quade \& O. Matthei resultados no publicados). 
en el resto del mundo, especialmente en Sudamérica esta evidencia sólo ha sido descubierta y utilizada en forma reciente. Asimismo, en el hemisferio Norte los estudios de dichas acumulaciones han carecido de diseño estructurado y de planificación organizada. Ellos han dependido fundamentalmente de la disponibilidad de fondos generada por proyectos específicos, realizados por investigadores asociados a regiones biogeográficas específicas. Sólo recientemente se han establecido bases de datos globales y comunes, las cuales pueden ser utilizadas para registrar y mapear la paleovegetación ${ }^{(2)}$, validar Modelos de Circulación Global (e.g., Bartlein et al. 1998), o establecer límites temporales a la dinámica sucesional matorral-pastizal. No existe sin embargo, un archivo central que permita extraer restos vegetales ya datados e identificados, los cuales constituyen fuente de información exquisita para estudios de tipo morfológicos (Van de Water et al. 1994), geoquímicos (Marino et al. 1992, Van de Water et al. 1994), y evolutivos (Smith et al. 1995).

El estudio de los depósitos fósiles en Norteamérica ha procedido sin un diseño que permita el desarrollo de investigación integrada y regional, o que anticipe la irrupción de nuevas técnicas como la extracción, amplificación y secuenciación de ADN antiguo, o la reconstrucción de cambios en la eficiencia del uso del agua a partir del análisis de isótopos de carbón en la celulosa de plantas fósiles. La experiencia acumulada en estudios similares de otras latitudes (e.g., Pearson \& Betancourt en prensa), en conjunto con el gran potencial de comunicación disponible en la actualidad para los investigadores, debería ser utilizada en favor del desarrollo de los estudios de paleomadrigueras de roedores en Sudamérica. Idealmente ellas deberían incluir una planificación adecuada de costo-beneficio que sobrepase los límites políticos e institucionales, integrando a todos los interesados en el estudio de las zonas áridas en Sudamérica. Ello permitiría establecer planes de desarrollo integrados entre las zonas áridas comunes en la región, impulsando de manera efectiva todas las áreas del conocimiento asociadas directa o indirectamente a las paleomadrigueras de roedores sudamericanos.

Finalmente, las paleomadrigueras ofrecen una oportunidad única para comparar la historia de la

\footnotetext{
(2) Véase http://climchange.cr.usgs.gov/data/ midden, http://www.nbs.gov/luhna/southwest/ southwest.html y http://geochange.er.usgs.gov/sw/ impacts/biology/veg_chg_model.
}

vegetación, así como la dinámica de largo plazo entre zonas áridas y semiáridas de Sudamérica y el resto del planeta. En los años 70, las similitudes entre los desiertos de Sudamérica (e.g., Monte) y Norteamérica (e.g., Sonora), sirvieron en parte de inspiración para el desarrollo de programas de investigación como el análisis de la estructura de ecosistemas, subprograma del IBP (Blair 1977, Mabry et al. 1977, Mooney 1977, Orians \& Solbrig 1977, Simpson 1977, Mares et al. 1985, Arroyo et al. 1994). A pesar de la incorporación del rol que el clima, la historia de la vegetación, e incluso el impacto humano, los cuales habrían afectado la estructura y organización de dichas comunidades (e.g., Bahre 1979), el IBP nunca incorporó el componente paleoecológico. Ello debido seguramente a la dificultad para obtener la evidencia necesaria para realizar dichos análisis. Dada la gran cantidad de información paleoecológica existente en Norteamérica, el desarrollo de investigaciones similares en otras zonas áridas del planeta, así como la perspectiva de desarrollo similar en Sudamérica, anticipamos un próspero futuro para las comparaciones interhemisféricas de paleovegetación y paleoclima entre dichas zonas.

\section{AGRADECIMIENTOS}

El trabajo de J.L.B. en paleomadrigueras de roedores Sudamericanos ha sido apoyado por una beca Fulbright en 1994, Proyecto I-033 del Instituto Inter Americano a la University of Arizona en 1996-1999, National Geographic Society en 1998 y 1999, National Science Foundation en 1999-2001 y por el Global Change Program del United States Geological Survey. Bárbara Saavedra es becaria doctoral de CONICYT (Comisión Nacional de Ciencia y Tecnología). Este artículo fue desarrollado durante la estadía de B.S. en la University of Arizona, como parte de un programa de intercambio entre University of Arizona y la Universidad de Chile, financiado por un proyecto U.S. AID, "Development of Sustainable Agriculture in Arid Regions of Chile," a B. Timmermann, University of Arizona y M. Arroyo, Universidad de Chile. Bárbara Saavedra agradece el apoyo brindado por los proyectos FONDECYT 1960930 y 2990120 . Julio Betancourt agradece la participación activa de las siguientes personas en los muestreos de paleomadrigueras en Sudamérica: Vera Markgraf, INSTAAR-University of Colorado, E. Cerdeño, M. Dakar, S. Monge, A. Ripalta, F. Roig, S. Roig, P. Villagra, y Wolfgang Volkheimer del CRICYTCONICET en Mendoza (Argentina); H. Lagiglia, 
G. Neme, A. Gil del Museo Municipal de Historia Natural en San Rafael; M. Christie de la Sociedad Naturalista Andino Patagónica en San Carlos de Bariloche (Argentina); C. Boero, y P. Ortiz de la Facultad de Ciencias Naturales e Instituto Miguel Lillo, Tucumán; Laura Scafati del Museo Argentino de Ciencias Naturales "Bernardino Rivadavia" en Buenos Aires (Argentina); A. Sbriller del INTA en San Carlos de Bariloche. Jaime Argollo, R. López, Clea Paz, R. Ríos, J. Vargas, de la Universidad Mayor de San Andrés en La Paz (Bolivia); Julio César Salinas del ORSTOM en La Paz. Mary T.K. Arroyo, C. Latorre, A. Maldonado, C. Villagrán de la Universidad de Chile en Santiago (Chile); P. Marquet y H. Samaniego de la P. Universidad Católica de Chile; L. Núñez de la Universidad Católica del Norte en Antofagasta (Chile). Vera Markgraf de la University of Colorado en Boulder, y a K. Aasen-Rylander del United States Geological Survey en Tucson (U.S.A.).

\section{LITERATURA CITADA}

ANDERSON RS \& TR VAN DEVENDER (1991) Comparison of pollen and macrofossils in packrat (Neotoma) middens: a chronological sequence from the Waterman Mountains, southern Arizona, USA. Review of Paleobotany and Palynology 68: 1-28.

ANDERSON RS \& TR VAN DEVENDER (1995) Vegetation history and paleoclimates of the coastal lowlands of Sonora, Mexico-pollen records from packrat middens. Journal of Arid Environments 30: 295-306

ANDERSON S (1997) Mammals of Bolivia, taxonomy and distribution. Bulletin of the American Museum of Natural History 231: 1-625.

ARAVENA L, C ASENJO \& A KIESSLING (1989) Claves para la identificación de pelos de mamíferos chilenos mediante secciones transversales. Seminario de Título en Biología y Ciencias Naturales, Instituto Profesional de Osorno, Osorno. 125 pp.

ARROYO MTK, FA SQUEO, JJ ARMESTO \& C VILLAGRÁN (1988) Effects of aridity on plant diversity in the northern Chilean Andes: results of a natural experiment. Annals of the Missouri Botanical Garden 75: 55-78.

ARROYO MTK, PH ZEDLER \& MD FOX (1994) Ecology and biogeography of Mediterranean ecosystems in Chile, California and Australia. Springer-Verlag, New York, New York. 455 pp.

BAHRE CJ (1979) Destruction of the natural vegetation of north-central Chile. University of California Publications in Geography 23: 1-117.

BARCENA JR \& FA ROIG (1982) Investigaciones arqueológicas en el área puneña de Mendoza, con especial referencia a Tephrocactus andicola (Cactaceae) como nuevo recurso alimentario. Relaciones de la Sociedad Argentina de Antropología XIV: 85-107.
BARTLEIN PJ, KH ANDERSON, PM ANDERSON, ME EDWARDS, CJ MOCK, RS THOMPSON, RS WEBB, $\mathrm{T}$ WEBB \& C WHITLOCK (1998) Paleoclimate simulations for North America over the past 21,000 years: features of the simulated climate and comparison with paleoenvironmental data. Quaternary Science Reviews 17: 549-585.

BERGER A \& MF LOUTRE (1991) Insolation values for the climate of the last 10 million years. Quaternary Science Reviews 10: 297-317.

BERRY SL (1991) The potential of fossil mammal middens as indicators of vegetation history in central Australia. Australian Journal of Botany 39: 305-313.

BETANCOURT JL (1984) Late Quaternary plant zonation and climate in southeastern Utah. Great Basin Naturalist 44: 1-35.

BETANCOURT JL \& TR VAN DEVENDER (1981) Holocene vegetation in Chaco Canyon, New Mexico. Science 214: 656-658.

BETANCOURT JL \& OK DAVIS (1984) Packrat middens from Canyon de Chelly, northeastern Arizona: paleoecological and archaeological considerations. Quaternary Research 21: 56-64.

BETANCOURT JL, A LONG, D DONAHUE, AJT JULL \& T ZABEL (1984) Pre-Columbian age for North American Corispermum L. (Chenopodiaceae) confirmed by accelerator radiocarbon dating. Nature 311: 653-656.

BETANCOURT JL, TR VAN DEVENDER \& M ROSE (1986) Comparison of plant macrofossils in woodrat (Neotoma sp.) and porcupine (Erethizon dorsatum) middens from the western United States. Journal of Mammalogy 67: 266-273.

BETANCOURT JL, TR VAN DEVENDER \& PS MARTIN (1990a) Packrat middens. The last 40,000 years of biotic change. The University of Arizona Press, Tucson, Arizona. 467 pp.

BETANCOURT JL, TR VAN DEVENDER \& PS MARTIN (1990b) Synthesis and prospectus. En: Betancourt JL, TR Van Devender \& P Martin (eds) Packrat middens: the last 40,000 years of biotic change: 435447. Arizona University Press, Tucson, Arizona.

BETANCOURT JL, WS SCHUSTER, JB MITTON \& RS ANDERSON (1991) Fossil and genetic history of a Pinyon pine (Pinus edulis) isolate. Ecology 72: 16851697.

BETANCOURT JL, C LATORRE, JA RECH, J QUADE \& KA RYLANDER (2000a) A 22,000-year record of monsoonal precipitation from northern Chile's Atacama desert. Science 289: 1542-1546.

BETANCOURT JL, KA RYLANDER, C PEÑALBA \& JL MCVICKAR (2000b) Late Quaternary vegetation history of Rough Canyon, south-central New Mexico, USA. Palaeogeography, Palaeoclimatology \& Palaeoecology 165: 71-95.

BINFORD MW, AL KOLATA, M BRENNER, JW JANUSEK, M SEDDON \& M ABBOTT (1997) Climate variation and the rise and fall of an Andean civilization. Quaternary Research 47: 235-248.

BLAIR WF (1977) Big biology: US/IBP. Dowden, Hutchison \& Ross, Stroudsburg, Pennsylvania. 261 pp. 
BOZINOVIC F \& LC CONTRERAS (1990) Basal rate of metabolism and temperature regulation of two desert herbivorous rodents: Octomys mimax and Tympanoctomys barrerae. Oecologia 84: 567-570.

BRAUN JK \& MA MARES (1996) Observations on unusual morphological and behavioral traits in Abrocoma (Rodentia: Abrocomidae) from Argentina. Journal of Mammalogy 77: 891-897.

BROECKER WS (1998) Paleoocean circulation during the last deglaciation: a bipolar seesaw? Paleoceanography 13: 119-121.

CARRION JS, L SCOTT \& JC VOGEL (1999) Twentieth century changes in montane vegetation in the eastern Free State, South Africa, derived from palynology of hyrax dung middens. Journal of Quaternary Science 14: $1-16$.

CINNAMON S \& R HEVLY (1988) Late Wisconsin macroscopic remains of Pinyon pine on the southern Colorado Plateau. Current Research in the Pleistocene 5: 47-48.

COLE KL (1982) Late Quaternary zonation of vegetation in the eastern Grand Canyon. Science 217: 11421145 .

COLE KL (1983) Late Pleistocene vegetation of Kings Canyon, Sierra Nevada, California. Quaternary Research 19: 117-129.

COLE KL (1985) Past rates of change, species richness, and a model of vegetational inertia in the Grand Canyon, Arizona. American Naturalist 125: 289-303.

COLE KL (1986) The lower Colorado River valley: a Pleistocene desert. Quaternary Research 25: 392-400.

COLE KL (1990) Reconstruction of past desert vegetation along the Colorado River using packrat middens. Palaeogeography, Palaeoclimatology \& Palaeoecology 76: 349-366.

COLE KL \& L MEYER (1982) Use of packrat middens to determine rates of cliff retreat in the eastern Grand Canyon, Arizona. Geology 10: 597-599.

COLE KL \& RH WEBB (1985) Late Holocene vegetation changes in Greenwater Valley, Mojave Desert, California. Quaternary Research 23: 227-235.

CRISCI JV, MM CIGLIANO, JJ MORRONE \& S ROIGJUÑENT (1991) Historical biogeography of southern South America. Systematic Zoology 40: 152-171.

D'ANTONI HL (1983) Pollen analysis of Gruta del Indio. Quaternary of South America and Antarctic Peninsula 1: $83-104$.

DAVIS OK (1990) Caves as sources of biotic remains in arid western North America. Palaeogeography, Palaeoclimatology \& Palaeoecology 76: 331-348.

ELIAS SA (1992) Late Quaternary zoogeography of the Chihuahuan Desert insect fauna, based on fossil records from packrat middens. Journal of Biogeography 19: 285-297.

ELIAS SA \& TR VAN DEVENDER (1990) Fossil insect evidence for late Quaternary climatic change in the Big Bend region, Chihuahuan Desert, Texas. Quaternary Research 34: 249-261.

ELIAS SA \& TR VAN DEVENDER (1992) Insect fossil evidence of late Quaternary environments in the northern Chihuahuan Desert of Texas and New Mexico: comparisons with the paleobotanical record. Southwestern Naturalist 37: 101-116.
ELIAS SA, TR VAN DEVENDER \& R DE BACA (1995) Insect fossil evidence of late glacial and Holocene environments in the Bolson de Mapimi, Chihuahuan Desert, Mexico: comparisons with the paleobotanical record. Palaios 10: 454-464.

FALL PL (1990) Deforestation in South Jordan: evidence from fossil hyrax middens. En: Bottema S, G EntjesNieborg \& W Van Zeist (eds) Man's role in the shaping of the eastern Mediterranean landscape: 271 281. A. Balkema, Rotterdam, The Netherlands.

FALL PL, CA LINDQUIST \& SE FALCONER (1990) Fossil hyrax middens from de Middle East: a record of paleovegetation and human disturbance. En: Betancourt JL, TR Van Devender \& P Martin (eds) Packrat middens: the last 40,000 years of biotic change: 408-427. University of Arizona Press, Tucson, Arizona.

FERNÁNDEZ J (1994) Cronología ${ }^{14} \mathrm{C}$ de los primeros estercoleros fósiles de la rata rupícola choschori (Octodontomys gliroides) de la Puna Argentina. Revista del Museo de Historia Natural de San Rafael (Mendoza) XII: 173-174.

FERNÁNDEZ J \& HO PANARELLO (1994) Ecología isotópica $\left(\delta^{13} \mathrm{C}\right)$ de dos variedades rupícola y campestre de ratas choschori (Octodontomys sp.) de la Puna Jujeña, República Argentina. Revista del Museo de Historia Natural de San Rafael (Mendoza) XII: $175-176$.

FINLEY RB (1990) Woodrat ecology and behavior and the interpretation of Paleomiddens. En: Betancourt JL, TR Van Devender \& P Martin (eds) Packrat middens. The last 40,000 years of biotic change: $28-42$. The University of Arizona Press, Tucson, Arizona.

GREEN N, J CALDWELL, J HOPE \& J LULY (1983) Pollen from a 1,800-year old stick-nest rat (Leporillus sp.) midden from Gnalta, western New South Wales. Quaternary Australasia 1: 31-41.

GROSJEAN M (1994) Paleohydrology of the Laguna Lejía (north Chilean Altiplano) and climatic implications for late-glacial times. Palaeogeography, Palaeoclimatology \& Palaeoecology 109: 271-286.

GROSJEAN M, MA GEYH, B MESSERLI \& U SCHOTTERER (1995) Late-glacial and early Holocene lake sediments, groundwater formation and climate in the Atacama altiplano $22-24^{\circ} \mathrm{S}$. Journal of Paleolimnology 14: 241-252.

HALL WE, TR VAN DEVENDER \& CA OLSON (1990) Arthropod history of the Puerto Blanco mountains, Organ Pipe National Monument, Southwestern Arizona. En: Betancourt JL, TR Van Devender \& P Martin (eds) Packrat middens: the last 40,000 years of biotic change: 363-379. University of Arizona Press, Tucson, Arizona.

HANSEN BS, HE WRIGHT JR \& JP BRADBURY (1984) Pollen studies in the Junin area, central Peruvian Andes. Geological Society of America Bulletin 95: 1454-1465.

HEUSSER CJ (1983) Quaternary pollen record from Laguna de Tagua Tagua, Chile. Science 219: 14291431.

HEUSSER CJ (1993) Late Glacial of southern South America. Quaternary Science Reviews 12: 345-350. 
HOLMGREN CA, JL BETANCOURT, KA RYLANDER, J ROQUE, O TOVAR, H ZEBALLOS, E LINARES \& J QUADE (2001) Holocene vegetation history from fossil rodent middens near Arequipa, Peru. Quaternary Research 56: 242-251.

HUNTER KL \& J MCAULIFFE (1994) Elevational shifts of Colyogene ramossissima in the Mojave Desert during the Little Ice Age. Quaternary Research 42: 216-221.

HUNTER KL, BR RIDDLE, JL BETANCOURT, KL COLE, TR VAN DEVENDER \& WG SPAULDING (2001) Ploidy race distributions since the Last Glacial Maximum in the North American desert shrub, Larrea tridentata. Global Ecology and Biogeography 10: 521-533.

JENNINGS SA \& DL ELLIOTT-FISK (1993) Packrat midden evidence of late Quaternary vegetation change in the White Mountains, California-Nevada. Quaternary Research 39: 214-221.

KEEFER DK, SD DEFRANC, ME MOSELEY, JB RICHARDSON III, D R SATTERLEE \& A DAYLEWIS (1998) Early maritime economy and El Niño events at Quebrada Tacahuay, Peru. Science 28: 1833-1835.

KING TJ (1976) Late Pleistocene-early Holocene history of coniferous woodland in the Lucerne Valley region, Mojave Desert, California. Great Basin Naturalist 36: 227-238.

KOEHLER PK \& RS ANDERSON (1994) Full-glacial shoreline vegetation during the maximum highstand at Owens Lake, California. Great Basin Naturalist 54: 142-149.

KOEHLER PK \& RS ANDERSON (1995) Thirty thousand years of vegetation changes in the Alabama Hills, Owens Valley, California. Quaternary Research 48: 238-248.

LANNER RM \& TR VAN DEVENDER (1974) Morphology of pinyons pine needles from fossil packrat middens in Arizona. Forest Science 20: 207-211.

LANNER RM \& TR VAN DEVENDER (1981) Late Pleistocene piñon pines in the Chihuahuan Desert. Quaternary Research 15: 278-290.

LANNER RM \& TR VAN DEVENDER (1998) The recent history of pines in the American Southwest. En: Richardson DM (ed) The ecology and biogeography of Pinus: 171-182. Cambridge University Press, Cambridge, United Kingdom.

LATORRE C, JL BETANCOURT, KA RYLANDER \& J QUADE (en prensa) Vegetation invasions into the Absolute Desert: a 45,000-yr rodent midden record from the Calama-Salar de Atacama Basin, northern Chile $\left(22-24^{\circ} \mathrm{S}\right)$. Geological Society of America Bulletin 114.

LESKINEN PH (1975) Occurrence of oaks in late Pleistocene vegetation in the Mojave Desert of Nevada. Madroño 23: 234-235.

LONG A, MARTIN PS \& LAGIGLIA H (1998) Ground sloth extinction and human occupation: Gruta del Indio. Radiocarbon 40: 693-281.

LONG A, LA WARNEKE, JL BETANCOURT \& RS THOMPSON (1990) Deuterium variations in plant cellulose from fossil packrat middens. En: Betancourt JL, TR Van Devender \& P Martin (eds) Packrat middens: the last 40,000 years of biotic change: 380 396. University of Arizona Press, Tucson, Arizona.
LOWENSTEIN JM, WN RAINEY \& JL BETANCOURT (1991) Immunospecific albumin in fossil packrat, porcupine and hyrax urine. Naturwissenschaften 78 : 26-27.

MABRY TJ, JH HUNZIKER \& DR DIFEO (1977) Creosote bush: biology and chemistry of Larrea in New World Deserts. Downden, Hutchinson and Ross, Strasbourg, Pennsylvania. 284 pp.

MARES MA, J MORELLO \& G GOLDSTEIN (1985) The Monte Desert and other subtropical semi-arid biomes of Argentina, with comments on their relation to North American arid areas. En: Evenari M, I NoyMeir \& DW Goodall (eds) Hot deserts and arid shrublands: 203-237. Elsevier Science Publishers B.V., Amsterdam, The Netherlands.

MARINO BD, MB MCELROY, RJ SALAWITCH \& WG SPAULDING (1992) Glacial-to interglacial variations in the carbon isotopic composition of atmospheric $\mathrm{CO}_{2}$. Nature 357: 461-464.

MARKGRAF V (1983) Late and postglacial vegetational and paleoclimatic changes in subantarctic, temperate, and arid environments in Argentina. Palynology 7: 43-70.

MARKGRAF V (1987) Paleoenvironmental changes at the northern limit of the subantarctic Nothofagus forest, Lat. $37^{\circ} \mathrm{S}$, Argentina. Quaternary Research 28: 119-129.

MARKGRAF V (1993) Younger Dryas in southernmost South America-an update. Quaternary Science Reviews 12: 351-355.

MARKGRAF V (1998) Researchers investigate interhemispheric climate linkages in the Americas and their societal effects. EOS: Transactions of the American Geophysical Union 79: 371-378.

MARKGRAF V, JL BETANCOURT \& KA RYLANDER (1997) Late-Holocene rodent middens from Río Limay, Neuquén Province, Argentina. The Holocene 7: 325-329.

MARQUET PA, LC CONTRERAS, S SILVA, JC TORRES-MURA \& F BOZINOVIC (1993) Natural history of Microcavia niata in the high Andean zone of northern Chile. Journal of Mammalogy 74: 136140.

MARTÍNEZ E (1984) El incendio de la vegetación en la Precordillera Mendocina III: Los pastizales disclimáxicos en la Quebrada de Villavicencio. Parodiana 3: 175-183.

McAULIFFE J \& TR VAN DEVENDER (1998) A 22,000yr record of vegetation change in the north-central Sonoran Desert. Palaeogeography, Palaeoclimatology \& Palaeoecology 141: 253-275.

MEAD JI \& AM PHILLIPS III (1981) The late Pleistocene and Holocene fauna and flora of Vulture Cave, Grand Canyon, Arizona. Southwestern Naturalist 26: $257-$ 288.

MEAD JI, TR VAN DEVENDER \& KL COLE (1983) Late Quaternary small mammals from Sonoran Desert packrat middens, Arizona and California. Journal of Mammalogy 64: 173-180.

MEHRINGER PJ \& CW FERGUSON (1969) Pluvial occurrence of bristlecone pine, Pinus aristata, in a Mohave Desert Mountain Range. Journal of the Arizona Academy of Science 5: 284-292. 
MOONEY HA (1977) Convergent evolution in Chile and California mediterranean climatic ecosystems. Dowden, Hutchinson \& Ross, Stroudsburg, Pennsylvania. 224 pp.

MORENO PI (1997) Vegetation and climate near Lago Llanquihue in the Chilean Lake District between 20,200 and 9,500 ${ }^{14} \mathrm{C}$ yr. B.P. Journal of Quaternary Science 12: 485-500.

MORENO PI, C VILLAGRÁN, PA MARQUET \& LG MARSHALL (1994) Quaternary Paleobiogeography of Northern and Central Chile. Revista Chilena de Historia Natural 67: 487-502.

NELSON DJ, RH WEBB \& A LONG (1990) Analysis of stick-nest rat (Leporillus sp.) middens from central Australia. En: Betancourt JL, TR Van Devender \& P Martin (eds) Packrat middens: the Last 40,000 Years of biotic change: 428-434. University of Arizona Press, Tucson, Arizona.

NORES M \& MM CERANA (1990) Biogeography of forest relics in the mountains of northwestern Argentina. Revista Chilena de Historia Natural 63: 37-46.

NORTHINGTON DK, ED MOREY \& TR VAN DEVENDER (1977) Phyletic interpretations from fossil (ca. 16,000 B.P.) remains of Sophora in the southwestern United States. Southwestern Naturalist 22: 495-504.

NOWAK RM \& JL PARADISO (1983) Walker's mammals of the world. The Johns Hopkins University Press, Baltimore, Maryland.

NOWAK CL, RS NOWAK, RJ TAUSCH, \& PE WIGAND (1994) Tree and shrub dynamics in northwestern Great Basin woodland and shrub steppe during the late Pleistocene and Holocene. American Journal of Botany 81: 265-277.

NÚÑEZ L (1983) Paleoindian and archaic cultural periods in the arid and semiarid regions of northern Chile. Advances in World Archaeology 2: 161-203.

NÚÑEZ LA (1994) The western part of South America (southern Peru, Bolivia, north-west Argentina and Chile) during the Stone age. En: Laet SJD (ed) History of humanity: 348-362. UNESCO.

OJEDA RA \& MA MARES (1989) A biogeographic analysis of the mammals of Salta province, Argentina. Special Publication of the Museum 27. Texas University Press, Lubbock, Texas.

ORIANS GH \& OT SOLBRIG (1977) Convergent evolution in warm deserts. Dowden, Hutchinson and Ross, Stroudsburg, Pennsylvania. 333 pp.

PEARSON OP \& MI CHRISTIE (1993) Rodent guano (amberat) from caves in Argentina. Studies on Neotropical Fauna and Environment 28: 105-111.

PEARSON S \& JR DODSON (1993) Stick-nest rat middens as sources of paleoecological data in Australian deserts. Quaternary Research 39: 347-354.

PEARSON $\mathrm{S} \& \mathrm{JL}$ BETANCOURT (en prensa) Understanding arid environments using fossil rodent middens. Journal of Arid Environments.

PENDALL E, JL BETANCOURT \& SW LEAVITT (1999) Plaeoclimatic significance of $\delta D$ and $\delta^{13} \mathrm{C}$ values in piñon pine needles from packrat middens spanning the last 40,000 years. Palaeogeography, Palaeoclimatology \& Palaeoecology 147: 53-72.
PHILLIPS AM III \& TR VAN DEVENDER (1974) Pleistocene packrat middens from the lower Grand Canyon of Arizona. Journal of the Arizona Academy of Science 9: 117-119.

PIANTANIDA MJ \& AM PETRIELLA (1976) Estudio morfológico de los pelos de algunas especies de roedores de la Provincia de Buenos Aires con el microscopio electrónico de barrido. Physis Sección C (Argentina) 35: 105-124.

PLUMMER MA, FM PHILLIPS, J FABRYKA-MARTIN, HJ TURIN, PE WIGN \& P SHARMA (1997) Chlorine36 in fossil rat urine: an archive of cosmogenic nuclide deposition during the past 40,000 years. Science 277 : 538-541.

POINAR HN, M HOFREITER, GW SPAULDING, PS MARTIN, BA STANKIEWICZ, H BLAND, RP EVERSHED, G POSSNERT \& S PÄÄBO (1998) Molecular coproscopy: dung and diet of the extinct ground sloth Nothrotheriops shastensis. Science 281: 402-406.

REDFORD KH \& JF EISENBERG (1992) Mammals of the Neotropics: the southern cone. Volume 2. Chile, Argentina, Uruguay, Paraguay. The University of Chicago Press, Chicago, Illinois.

RHINEHART RB \& DA MCFARLANE (1995) Early Holocene vegetation record from the Salton Basin. Quaternary Research 43: 259-262.

RHODE (2001) Packrat middens as a tool for reconstructing historic ecosystems. En: Egan D \& E Howell (eds) Historical ecology handbook: a restorationist's guide to reference ecosystems: 257-293. Island Press, Washington, District of Columbia. $457 \mathrm{pp}$.

RHODE D \& DB MADSEN (1995) Late Wisconsin/Early Holocene vegetation in the Bonneville Basin. Quaternary Research 44: 246-256.

ROGERS SO \& AJ BENDICH (1985) Extraction of DNA milligram amounts of fresh, herbarium and mummified plant tissues. Plant Molecular Biology 5: 69-76.

ROIG FA \& JA AMBROSSETTI (1971) Investigaciones climáticas I. Restos de un estrato arbóreo bajo de Schinus polygamus en la precordillera de Mendoza. Deserta II: 125-130.

ROIG JS (1994) Historia biogeográfica de América del Sur Austral. Multequina 3: 167-203.

SANDWEISS DH, H MCINNIS, RL BURGER, A CANO, B OJEDA, R PAREDES, MC SANDWEISS \& MD GLASCOCK (1998) Quebrada Jaguay: early South American maritime adaptations. Science 281: 18301832.

SCOTT L \& JC VOGEL (1992) Short-term changes of climate and vegetation revealed by pollen analysis of hyrax dung in South Africa. Review of Palaeobotany and Palynology 74: 283-291.

SIMPSON BB (1977) Mesquite: its biology in two desert ecosystems. Dowden, Hutchinson \& Ross, Stroudsburg, Pennsylvania. $250 \mathrm{pp}$.

SMITH FA \& JL BETANCOURT (1998) Response of bushy-tailed woodrats (Neotoma cinerea) to Late Quaternary climatic change in the Colorado Plateau. Quaternary Research 50: 1-11.

SMITH FA, JL BETANCOURT \& JH BROWN (1995) Evolution of body size in the woodrat over the past 25,000 years of climate change. Science 270: 20122014. 
SPAULDING WG (1977) Late Quaternary vegetation change in the Sheep Range, southern Nevada. Journal of the Arizona Academy of Sciences 12: 3-8.

SPAULDING WG (1983) Late Wisconsin macrofossil records of desert vegetation in the American Southwest. Quaternary Research 19: 256-264.

SPAULDING WG (1985) Vegetation and climates of the last 45,000 years in the vecinity of the Nevada Test Site, south-central Nevada. U.S. Geological Survey Professional Paper 1329. 199 pp.

SPAULDING WG \& LJ GRAUMLICH (1986) The last pluvial climatic episodes in the deserts of southwestern North America. Nature 320: 541-544.

SPAULDING WG, EB LEOPOLD \& TR VAN DEVENDER (1983) Late Wisconsin paleoecology of the American Southwest. En: Porter SC (ed) Late Quaternary environments of the United States. Volume 1, The late Pleistocene: 259-293. University of Minnesota Press, Minneapolis, Minnesota.

SPAULDING WG, JL BETANCOURT, LK CROFT \& KL COLE (1990) Packrat middens: their composition and methods of analysis. En: Betancourt JL, TR Van Devender \& P Martin (eds) Packrat middens: the last 40,000 years of biotic change: 59-84. University of Arizona Press, Tucson, Arizona.

STEPPAN SJ (1998) Phylogenetic relationships and species limits within Phyllotis (Rodentia: Sigmodontinae): concordance between mtDNA sequence and morphology. Journal of Mammalogy 79: 573-593.

SWETNAM TW, CD ALLEN \& JL BETANCOURT (1999) Applied historical ecology: using the past to manage the future. Ecological Applications 9: 1189-1206.

THINON M, A BALLOUCHE \& M REILLE (1996) Holocene vegetation of the central Saharan mountains: the end of a myth. The Holocene 6: 457-462.

THOMPSON RS (1979) Late Pleistocene and Holocene packrat middens from Smith Creek Canyon, White Pine County, Nevada. Nevada State Museum Anthropological Papers 17: 362-380.

THOMPSON RS (1985) Palynology and Neotoma middens. American Association Stratigraphic Palynologists, Contribution Series 16: 89-112.

THOMPSON RS (1992) Late Quaternary environments in Ruby Valley, Nevada. Quaternary Research 37: 1-15.

THOMPSON RS \& JI MEAD (1982) Late Quaternary environments and biogeography of the Great Basin. Quaternary Research 17: 39-55.

THOMPSON RS, C WHITLOCK, PJ BARTLEIN, SP HARRISON \& WG SPAULDING (1993) Climatic changes in the western United States since 18,000 yr B.P. En: Wright HE, J Kutzbach, T Webb, WF Ruddiman, FA Street-Perrott \& PJ Bartlein (eds) Global climates since the Last Glacial maximum: 468513. University of Minnesota Press, Minneapolis, Minnesota.

VALERO-GARCES BL, M GROSJEAN, A SCHALB, MA GEYH, B MESSERLI \& K KELTS (1996) Limnology of Laguna Miscanti: Evidence for mid to late Holocene moisture changes in the Atacama Altiplano (Northern Chile). Journal of Paleolimnology 16: 1-21.

VAN DEVENDER TR (1977) Holocene woodlands in the southwestern deserts. Science 198: 198-192.
VAN DEVENDER TR (1987) Holocene vegetation and climate in the Puerto Blanco Mountains, southwestern Arizona. Quaternary Research 27: 51-72.

VAN DEVENDER TR \& JE KING (1971) Late Pleistocene vegetation records in western Arizona. Journal of the Arizona Nevada Academy of Sciences 6: 240-244.

VAN DEVENDER TR \& JI MEAD (1976) Late Pleistocene and modern plant communities of Shinumo Creek and Peach Springs Wash, lower Grand Canyon, Arizona. Journal of the Arizona Academy of Sciences 11: 16-22.

VAN DEVENDER TR \& B EVERITT (1977) The latest Pleistocene and Recent vegetation of the Bishop's Cap, south-central New Mexico. Quaternary Research 22: 337-352.

VAN DEVENDER TR \& D RISKIND (1979) Late Pleistocene and early Holocene plant remains from Hueco Tanks State Historical Park: the development of a refugium. Southwestern Naturalist 24: 127-140.

VAN DEVENDER TR \& WG SPAULDING (1979) Development of vegetation and climate in the southwestern United States. Science 204: 701-710.

VAN DEVENDER TR \& TL BURGESS (1985) Late Pleistocene woodlands in the Bolson de Mapimi: a refugium for the Chihuahuan desert biota? Quaternary Research 24: 346-353.

VAN DEVENDER TR \& GL BRADLEY (1990) Late Quaternary mammals from the Chihuahuan desert: paleoecology and latitudinal gradients. En: Betancourt JL, TR Van Devender \& P Martin (eds) Packrat middens: the last 40,000 years of biotic changes: 350 362. University of Arizona Press, Tucson, Arizona.

VAN DEVENDER TR \& JF WIENS (1993) Holocene changes in the flora of Ragged Top, south-central Arizona. Madroño 40: 246-264.

VAN DEVENDER TR \& WE HALL (1994) Holocene arthropods from the Sierra Bacha, Sonora, Mexico, with emphasis on beetles. The Coleopterists Bulletin 48: 30-50.

VAN DEVENDER, CE FREEMAN \& RD WORTHINGTON (1978) Full-glacial and recent vegetation of the Livingston Hills, Presidio County, Texas. Southwestern Naturalist 23: 289-302.

VAN DEVENDER TR, JL BETANCOURT \& M WIMBERLY (1984) Biogeographic implications of a packrat midden sequence from the Sacramento Mountains, south-central New Mexico. Quaternary Research 22: 344-360.

VAN DEVENDER TR, PS MARTIN, RS THOMPSON, KL COLE, AJT JULL, A LONG, LJ TOOLIN \& DJ DONAHUE (1985) Fossil packrat middens and the tandem accelerator mass spectrometer. Nature 317 : 610-613.

VAN DEVENDER TR, RS THOMPSON \& JL BETANCOURT (1987) Vegetation history of the deserts of southwestern North America: the nature and timing of the late Wisconsin-Holocene transition. En: Ruddiman WF \& HEJ Wright (eds) North America and adjacent oceans during the last deglaciation, The Geology of North America: 323-352. Geological Society of America, Boulder, Colorado.

VAN DEVENDER TR, TL BURGESS, RS FELGER \& RM TURNER (1990) Holocene vegetation of the Hornaday Mountains. Proceedings of the San Diego Society of Natural History 2: 1-19. 
VAN DEVENDER TR, JI MEAD \& AM REA (1991) Quaternary plants and vertebrates from Picacho Peak, Arizona. Southwestern Naturalist 36: 302-314.

VAN DEVENDER TR, TL BURGESS, JC PIPER \& RM TURNER (1994) Paleoclimatic implications of Holocene plant remains from the Sierra Bacha, Sonora, Mexico. Quaternary Research 41: 99-108.

VAN DE WATER PK, SW LEAVITT \& JL BETANCOURT (1994) Trends in stomatal density and ${ }^{13} \mathrm{C} /{ }^{12} \mathrm{C}$ ratios of Pinus flexilis needles during last GlacialInterglacial cycle. Science 264: 239-243.

VILLA-MARTÍNEZ R \& C VILLAGRÁN (1997) Historia de la vegetación de bosques pantanosos de la costa de Chile central durante el Holoceno Medio y Tardío. Revista Chilena de Historia Natural 70: 391-401.

VILLAGRÁN C \& J VARELA (1990) Palynological evidence for increased aridity on the central Chilean coast during the Holocene. Quaternary Research 34: 198-207.

VILLAGRÁN C, MT KALIN ARROYO \& C MARTICORENA (1983) Efectos de la desertización en la distribución de la flora andina de Chile. Revista Chilena de Historia Natural 56: 137-157.

VUILLE M \& C AMMAIN (1997) Regional snowfall patterns in the high, arid Andes. Climatic Change 36: 413-423.

WELLS PV (1966) Late Pleistocene vegetation and degree of pluvial climatic change in the Chihuahuan Desert. Science 153: 970-975.

WELLS PV (1970) Postglacial vegetational history of the Great Plains. Science 167: 1574-1582.
WELLS PV (1976) Macrofossil analysis of woodrat (Neotoma) middens as a key to the Quaternary vegetational history of arid America. Quaternary Research 6: 223-248.

WELLS PV (1983) Paleogeography of montane islands in the Great Basin since the last glaciopluvial. Ecological Monographs 563: 341-382.

WELLS PV \& CV JORGENSEN (1964) Pleistocene wood rat middens and climatic change in Mohave Desert: a record of juniper woodlands. Science 143: 1171-1174.

WELLS PV \& R BERGER (1967) Late Pleistocene wood rat middens and climatic change in the Mohave Desert. Science 155: 1640-1647.

WELLS PV \& D WOODCOCK (1985) Full-glacial vegetation of Death Valley, California: Juniper woodland opening to Yucca semidesert. Madroño 32: 11-23.

WOODCOCK D (1986) The late Pleistocene of Death Valley: a climatic reconstruction based on macrofossil data. Palaeogeography, Palaeoclimatology \& Palaeoecology 57: 273-283.

WILSON DE \& DA REEDER (1993) Mammal species of the world: a taxonomic and geographic reference. Smithsonian Institution Press, Washington, District of Columbia.

YBERT JP (1992) Ancient lake environments as deduced from pollen analysis. En: Dejoux C \& A Iltis (eds) Lake Titicaca: a synthesis of limnological knowledge: 49-62. Kluwer Academic Publishers, Dordrecht, The Netherlands.

Editor Asociado: P. Marquet

Recibido el 24 de enero de 2002; aceptado el 29 de abril de 2002 